\title{
Analysis of finite microstrip structures using an efficient implementation of the integral equation technique
}

\author{
F. Quesada Pereira, J. L. Gomez Tornero, D. Cañete Rebenaque, \\ J. Pascual Garcia, and A. Alvarez Melcon \\ Department of Information Technologies and Communications, Technical University of Cartagena, Cartagena, Spain
}

Received 23 January 2004; revised 1 October 2004; accepted 26 October 2004; published 27 January 2005.

[1] An efficient numerical implementation of the integral equation technique (IE) has been developed for the analysis of the electrical characteristics of finite microstrip structures. The technique formulates a volume version of the IE for the finite dielectric objects and a standard surface IE technique for the metallic areas. The system of integral equations formulated is solved with special numerical techniques described in this paper. The input impedances of several microstrip antennas have been computed, showing good agreement with respect measurements. The technique has shown to be accurate, even for complex geometries containing several stacked dielectric layers. The radiation patterns of the structures have also been computed, and measured results from real manufactured hardware confirm that backside radiation and secondary lobes are accurately predicted by the theoretical model. The paper also discuss a suitable excitation model for finite size ground planes and investigates the possibilities for an independent meshing of the metallic areas and the dielectric objects inside a given geometry. The practical value of the approach derived is that microstrip circuits can be designed, minimizing the volume and size of the dielectric substrates.

Citation: Quesada Pereira, F., J. L. Gomez Tornero, D. Cañete Rebenaque, J. Pascual Garcia, and A. Alvarez Melcon (2005), Analysis of finite microstrip structures using an efficient implementation of the integral equation technique, Radio Sci., 40, RS1004, doi:10.1029/2004RS003036.

\section{Introduction}

[2] The study of microstrip structures is a subject that is attracting much attention in microwave engineering, specially for their mechanical advantages, and for the integration capabilities with other radio frequency circuits offered by these configurations. For the analysis of microstrip structures, the integral equation (IE) technique in combination with the multilayered media Green's functions formulated both in the spectral [Mesa and Marques, 1995] and in the spatial [Kinayman and Aksun, 1997] domain, has grown in popularity. Using this approach, the information of the dielectric layers is included in the Green's functions, so that the numerical treatment of the problem is reduced to the metallic areas printed on the substrates, thus considerably increasing the efficiency of the technique. A big limitation of this approach, however, is that the dielectric layers are

Copyright 2005 by the American Geophysical Union. 0048-6604/05/2004RS003036 considered to be of infinite transverse dimensions [Bunger and Arndt, 1997]. In addition, the infinite size analysis can not extract other important information, such as the backside radiation of printed microstrip antennas [Mosig and Gardiol, 1982]. A third limitation of this technique is that, while the analysis of strictly planar geometries is relatively easy [Mosig and Gardiol, 1982], the same is generally not true when vertical or arbitrarily oriented metalizations are included inside the dielectric layers [Michalski and Zheng, 1990; Kinayman and Aksun, 1997; Gay-Balmaz and Mosig, 1997]. Also the practical circuits must be of finite size. In order to obtain accurate results with respect to the infinite model, the size of the substrates must contain several wavelengths of the circuit, in order to approach to the ideal infinite size situation. A drawback of this design procedure is that the size and volume of the total circuit can not be conveniently optimized, which might not be acceptable for many satellite and space applications.

[3] The techniques that are readily available for the analysis of full 3-D finite microstrip structures are the 
finite elements, finite differences, or the method of lines. These techniques, however, require very intensive numerical computations, specially because the whole volume around the structure must be discretized, often in combination of some sort of absorbing boundary condition [Aksun and Dural, 1996]. As regards the integral equation technique, some previous attempts for the analysis of finite microstrip structures have been reported, both using volume and surface equivalent formulations [Sarkar and Arvas, 1990; Sarkar et al., 1990; Chew et al., 2001]. Also, in the work of $L u$ and Chew [2000], metallic areas coated with dielectric regions were analyzed with a volume integral equation formulation, in the context of the calculation of the scattering properties of such objects. However, only very preliminary results of very simple microstrip antenna structures have been presented in the work of Sarkar et al. [1990]. Also, in the work of Sarkar et al. [1990], the radiation patterns obtained with volume and surface formulations appear not to agree well, thus indicating limited accuracy in the presented results. A different but very interesting approach for extracting the radiation patterns is addressed in the work of Bokhari et al. [1992], where the induced currents on the metallic areas are first calculated assuming an infinite dielectric slab. The finite dimensions are later taken into account during the computation of the far field, by considering only vertical polarization currents. In addition, there are not many precise input impedance results of finite size microstrip structures reported in the literature [Tavakkol-Hamedani et al., 2002; Lu and $Y u, 2002]$.

[4] In this contribution we present two efficient numerical implementations of the volume equivalent integral equation technique applied to the analysis of finite microstrip structures. The technique combines rooftop basis and testing functions for the conductor areas, with two different choices for the finite dielectric objects. The first is based on combining pulse basis functions and deltas for testing, in a similar way as presented in the work of Sarkar et al. [1990]. Unlike the technique in the work of Sarkar et al. [1990], however, the use of rooftop basis and testing functions for the conductor areas increases the numerical stability of the method, and accurate results are shown to be obtained, both for the input impedance and for the radiation patterns of microstrip antennas. The second choice to treat the finite dielectric objects is a full Galerkin approach, which employs rooftop basis and testing functions defined in the volume of the brick cells used in the discretization of the dielectrics. This type of basis functions is similar to the one introduced in the work of Catedra et al. [1989] in the context of the radar cross-section computation of pure dielectric objects. In that work, however, one dimensional pulses were used for testing, and uniform

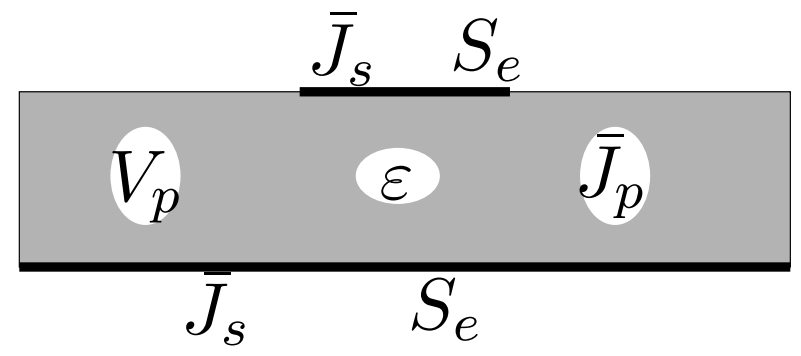

Figure 1. General finite size microstrip structure analyzed in this paper.

cells had to be used to employ the conjugate gradientfast Fourier transform method. The advantage of using rooftop basis functions is that the derivatives on the scalar potential Green's functions are avoided, therefore reducing considerably the level of singularity in the subsequent numerical treatment.

[5] In addition, a novel excitation model, which can be used with finite size ground planes, has also been derived. The advantage of this model is that the ground plane can be meshed independently from the input feeding line. Also, a novel numerical procedure has been developed to allow for the independent meshing of the dielectric bodies with respect to touching metallic areas of the circuit. All these novel numerical techniques add flexibility to the volume equivalent IE method. Theoretical results are compared with measurements indicating that the new numerical models are indeed accurate, and can be used for the analysis of real life microstrip antennas and printed circuits.

\section{Theoretical Outline}

[6] The formulation of a finite size microstrip structure as shown in Figure 1, using the volume equivalent integral equation technique, follows the considerations presented in the work of Sarkar et al. [1990]. For the metallic areas the boundary condition to impose is zero tangent electric field, namely:

$$
\bar{E}_{t}^{(\mathrm{tot})}=\left.\left[\bar{E}_{t}^{(\mathrm{exc})}+\bar{E}_{t}^{(\mathrm{scat})}\right]\right|_{S_{e}}=0
$$

In above equation, $\bar{E}_{t}^{(\mathrm{exc})}$ is the exciting electric field, which represents the excitation of the microstrip structure of Figure 1. In this work the circuit is excited with a coaxial probe, and a delta gap excitation model as described in the work of Eleftheriades and Mosig [1996] and Melcon et al. [1999] is used. In addition, $\bar{E}_{t}^{\text {(scat) }}$ is the scattered field, which for the microstrip structure of Figure 1 is due to both polarization 


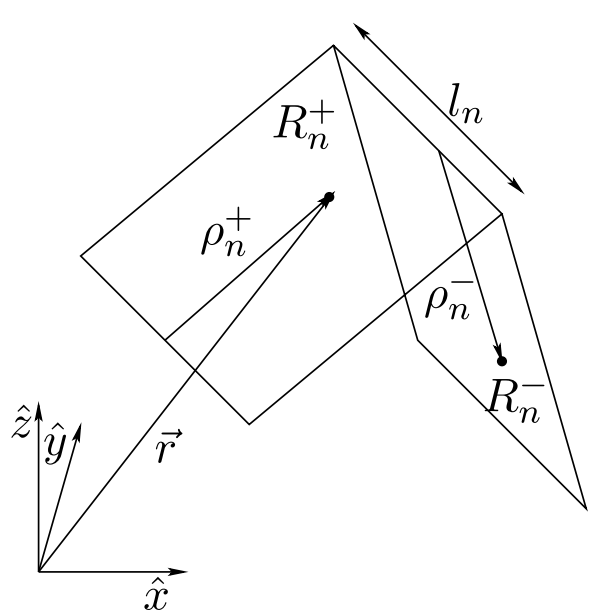

$$
\begin{gathered}
\bar{f}_{m}^{e}(\bar{r})=\bar{f}_{n}^{e}\left(\bar{r}^{\prime}\right)=\left\{\begin{array}{cl}
\frac{l_{n}}{A_{n}^{+}} \vec{\rho}_{n}^{+} & , \bar{r}^{\prime} \in R_{n}^{+} \\
\frac{l_{n}}{A_{n}^{-}} \vec{\rho}_{n}^{-} & , \bar{r}^{\prime} \in R_{n}^{-} \\
0 & , \text { Otherwise }
\end{array}\right. \\
\nabla_{s}^{\prime} \cdot \bar{f}_{n}^{e}\left(\bar{r}^{\prime}\right)=\left\{\begin{array}{cc}
\frac{l_{n}}{A_{n}^{+}} & , \bar{r}^{\prime} \in R_{n}^{+} \\
-\frac{l_{n}}{A_{n}^{-}} & , \bar{r}^{\prime} \in R_{n}^{-} \\
0 & , \text { Otherwise }
\end{array}\right.
\end{gathered}
$$

Figure 2. Basis and testing employed for the conducting areas.

currents on the dielectric and surface currents on the metallic areas:

$$
\bar{E}^{\text {(scat) }}=\int_{V_{p}} \overline{\bar{G}}_{E_{J}} \cdot \bar{J}_{p} d V^{\prime}+\int_{S_{e}} \overline{\bar{G}}_{E_{J}} \cdot \bar{J}_{s} d S^{\prime}
$$

where $\overline{\bar{G}}_{E_{J}}$ is the dyadic electric field Green's function produced by an electric current, $J_{p}$ are the polarization currents defined on the volume of the dielectric objects, and $J_{s}$ are the surface currents induced on the metallic areas of the structure. In the present work, the mixed potential Green's functions are used instead of the field Green's functions in order to reduce the singular behavior [Mosig, 1988]. Furthermore, in the dielectric region the volume equivalent imposes the following restriction on the total electric field:

$$
\bar{J}_{p}=\left.j \omega\left(\varepsilon-\varepsilon_{0}\right) \bar{E}^{(\mathrm{tot})}\right|_{V_{p}}
$$

If we use the delta gap excitation model [Eleftheriades and Mosig, 1996; Melcon et al., 1999], the exciting electric field $\bar{E}_{t}^{\text {(exc) }}$ only exist at the connector position contacting the exciting coaxial probe, and therefore it is zero inside the dielectric. Using (1) we can then reduce (3) as follows:

$$
\bar{J}_{p}=\left.j \omega\left(\varepsilon-\varepsilon_{0}\right) \bar{E}^{\text {(scat })}\right|_{V_{p}}
$$

Combining now equations (1), (2) and (4), the following system of coupled integral equations are easily obtained:

$$
\begin{aligned}
& \bar{E}_{t}^{(\mathrm{exc})}=\left\{-\int_{V_{p}} \overline{\bar{G}}_{E_{J}} \cdot \bar{J}_{p} d V^{\prime}-\int_{S_{e}} \overline{\bar{G}}_{E_{J}} \cdot \bar{J}_{S} d S^{\prime}\right\}_{S_{e}} \\
& 0=\left\{\frac{\bar{J}_{p}}{j \omega\left(\varepsilon-\varepsilon_{0}\right)}-\int_{V_{p}} \overline{\bar{G}}_{E_{J}} \cdot \bar{J}_{p} d V^{\prime}-\int_{S_{e}} \overline{\bar{G}}_{E_{J}} \cdot \bar{J}_{s} d S^{\prime}\right\}_{V_{p}}
\end{aligned}
$$

the solution of this system of integral equations gives the unknown polarization currents inside the dielectrics $\bar{J}_{p}$ and induced surface currents on the metallic areas $\bar{J}_{s}$. Once known, the electromagnetic fields in the structure can be easily recovered with (2).

\section{Numerical Implementation}

[7] For the numerical solution of (5), the surface of the metallic areas are always discretized using rooftop basis and testing functions defined in rectangular patches (see (6) and Figure 2).

$$
\bar{J}_{s}\left(\bar{r}^{\prime}\right)=\sum_{k=1}^{N_{e}} \alpha_{n}^{e} \bar{f}_{n}^{e}\left(\bar{r}^{\prime}\right)
$$

This is a good choice for representing the induced currents on the printed metallic areas, since it combines accuracy with an easy implementation [Mosig, 1988]. Higher-order basis functions can also be used, but complexity for implementation increases accordingly [Gedney and Lu, 2003]. In the present work, the rooftop selection has proven to lead to accurate results when obtaining the scattering parameters and input impedances of printed microstrip circuits.

[8] When solving the system of integral equation (5) with the method of moments (MoM), the conductorconductor interactions are always given by:

$$
\begin{aligned}
Z_{m, n}^{(e, e)}= & j \omega \int_{S_{e m}} \bar{f}_{m}^{e}(\bar{r}) d S \int_{S_{e n}} \bar{f}_{n}^{e}\left(\bar{r}^{\prime}\right) \overline{\bar{G}}_{A}\left(\bar{r}, \bar{r}^{\prime}\right) d S^{\prime} \\
& +\frac{1}{j \omega} \int_{S_{e m}} \nabla \cdot \bar{f}_{m}^{e}(\bar{r}) d S \int_{S_{e n}} \nabla^{\prime} \cdot \bar{f}_{n}^{e}\left(\bar{r}^{\prime}\right) G_{V}\left(\bar{r}, \bar{r}^{\prime}\right) \\
& \cdot d S^{\prime}
\end{aligned}
$$

where $\overline{\bar{G}}_{A}\left(\bar{r}, \bar{r}^{\prime}\right)$ is the free-space magnetic vector potential Green's function, and $G_{V}\left(\bar{r}, \bar{r}^{\prime}\right)$ is the freespace electric scalar potential Green's function. 


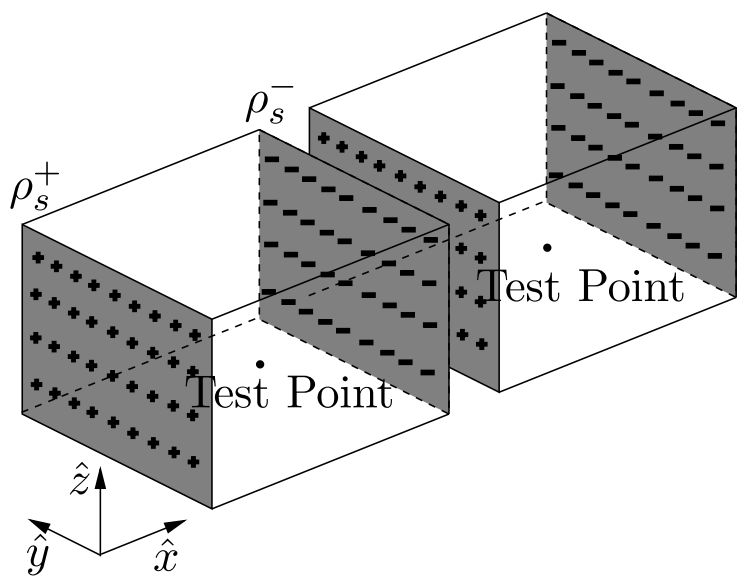

$$
\begin{aligned}
& \bar{f}_{n}^{d}\left(\bar{r}^{\prime}\right)=\left\{\begin{array}{cl}
\hat{e}_{n} & , \vec{r}^{\prime} \in B_{n} \\
0 & , \text { Otherwise }
\end{array}\right. \\
& \bar{f}_{m}^{d}(\bar{r})=\hat{e}_{m} \delta\left(\bar{r}_{m}-\bar{r}\right)
\end{aligned}
$$

Figure 3. Test point and fictitious surface charge density on a generic brick domain (rooftoppulse numerical implementation).

[9] For the discretization of the finite dielectric objects, a first choice has been presented in the work of Sarkar et al. [1990]. In this case a combination of pulse basis functions with deltas for testing, defined in rectangular prism, is selected (see (8) and Figure 3):

$$
\bar{J}_{p}\left(\bar{r}^{\prime}\right)=\sum_{k=1}^{N_{p}} \alpha_{n}^{d} \bar{f}_{n}^{d}\left(\bar{r}^{\prime}\right)
$$

[10] When applying the MoM technique, this formulation leads to a matrix system which is not symmetrical, since the testing procedure on the surface of the metallic areas is performed in a different way as the testing in the dielectric volume. The testing on the metallic areas is applied through a surface integration, namely:

$$
\begin{aligned}
Z_{m, n}^{(e, d)}= & j \omega \int_{S_{e}} \bar{f}_{m}^{e}(\bar{r}) d S \int_{V_{p}} \bar{f}_{n}^{d}\left(\bar{r}^{\prime}\right) \overline{\bar{G}}_{A}\left(\bar{r}, \bar{r}^{\prime}\right) d V^{\prime} \\
& +\frac{1}{j \omega} \int_{S_{e}} \nabla \cdot \bar{f}_{m}^{e}(\bar{r}) d S \int_{V_{p}} \nabla^{\prime} \cdot \bar{f}_{n}^{d}\left(\bar{r}^{\prime}\right) G_{V}\left(\bar{r}, \bar{r}^{\prime}\right) \\
& \cdot d V^{\prime}
\end{aligned}
$$

where $\bar{f}_{n}^{d}\left(\bar{r}^{\prime}\right)$ is the basis function in the dielectric, and $\bar{f}_{m}^{e}(\bar{r})$ is the test function on the metallic surface.

[11] In above expression, the divergence of the basis function leads to two surface charges on the corresponding sides of the cube, as shown in Figure 3. In this way the second volume integral is further decomposed into two surface integrals, extended to these two sides of the cube.

[12] However, for the dielectric object a testing using deltas is applied. In this case the matrix entry is reduced to:

$$
\begin{aligned}
Z_{m, n}^{(d, e)}= & j \omega \int_{S_{e}} \hat{e}_{m} \overline{\bar{G}}_{A}\left(\bar{r}, \bar{r}^{\prime}\right) \bar{f}_{n}^{e}\left(\bar{r}^{\prime}\right) d S^{\prime}-\frac{1}{j \omega} \int_{S_{e}} \hat{e}_{m} \\
& \cdot \nabla G_{V}\left(\bar{r}, \bar{r}^{\prime}\right) \nabla^{\prime} \cdot \bar{f}_{n}^{e}\left(\bar{r}^{\prime}\right) d S^{\prime}
\end{aligned}
$$

where $\bar{f}_{n}^{e}\left(\bar{r}^{\prime}\right)$ is the basis function on the metallic area, $\hat{e}_{m}$ is the direction of the delta testing function, and $\bar{r}_{m}$ is the point inside the volume of the dielectric where the delta function is applied.

[13] For the dielectric-dielectric interaction, a similar testing procedure is applied, thus resulting into:

$$
\begin{aligned}
Z_{m, n}^{(d, d)}= & \frac{\delta_{m n}}{j \omega\left(\varepsilon-\varepsilon_{0}\right)}+j \omega \int_{V_{p}} \hat{e}_{m} \overline{\bar{G}}_{A}\left(\bar{r}, \bar{r}^{\prime}\right) \bar{f}_{n}^{d}\left(\bar{r}^{\prime}\right) d V^{\prime} \\
& -\frac{1}{j \omega} \int_{V_{p}} \hat{e}_{m} \cdot \nabla G_{V}\left(\bar{r}, \bar{r}^{\prime}\right) \nabla^{\prime} \cdot \bar{f}_{n}^{d}\left(\bar{r}^{\prime}\right) d V^{\prime}
\end{aligned}
$$

where $\delta_{m n}$ refers to the Kronecker $\delta$ function. Once all these reaction integrals are computed, a system of linear equations is set up in the following way:

$$
\left[\begin{array}{c}
\bar{E}_{t}^{(\mathrm{exc})} \\
0
\end{array}\right]=\left[\begin{array}{ll}
Z^{e e} & Z^{d e} \\
Z^{e d} & Z^{d d}
\end{array}\right] \cdot\left[\begin{array}{c}
c \alpha^{e} \\
\alpha^{d}
\end{array}\right]
$$

[14] In particular, it can be observed in (10) that, when the base is taken in the metallic areas and the testing in the dielectric, the gradient of the electric scalar potential Green's function can not be avoided. An important difference between our implementation and the one presented in the work of Sarkar et al. [1990], is that we evaluate this gradient analytically, avoiding the numerical derivatives used in the work of Sarkar et al. [1990]. In any case, the reaction integrals contain a singularity of the order of $\left(1 / r^{2}\right)$, which is only integrable in the Cauchy sense, and can not be extracted easily with numerical procedures. To avoid the singularity, the testing using deltas is applied at the center point of the volume cell as shown in Figure 3. The advantage of doing so is that the testing point never lies inside the base integration, therefore avoiding the singularity of the Green's function. However, if the 


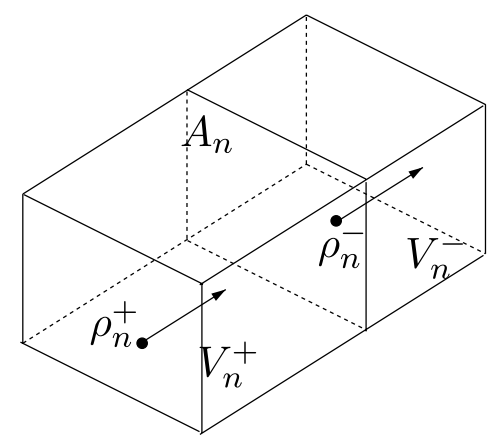

$$
\begin{gathered}
\bar{f}_{n}\left(\bar{r}^{\prime}\right)=\left\{\begin{array}{cc}
\frac{A_{n}}{V_{n}^{+}} \bar{\rho}_{n}^{+} & , \bar{r}^{\prime} \text { en } B_{n}^{+} \\
\frac{A_{n}}{V_{n}^{-}} \bar{\rho}_{n}^{-} & , \bar{r}^{\prime} \text { en } B_{n}^{-} \\
0 & , \text { Otherwise }
\end{array}\right. \\
\bar{\nabla} \cdot \bar{f}_{n}\left(\bar{r}^{\prime}\right)=\left\{\begin{array}{cc}
+\frac{A_{n}}{V_{n}^{+}} & , \bar{r}^{\prime} \text { en } B_{n}^{+} \\
-\frac{A_{n}}{V_{n}^{-}} & , \bar{r}^{\prime} \text { en } B_{n}^{-} \\
0 & , \text { Otherwise }
\end{array}\right.
\end{gathered}
$$

Figure 4. Basis functions defined in two contiguous volume brick cells.

thickness of the dielectric substrate is electrically very small, then a Green's function exhibiting fast variations must be integrated, since then the testing point lies very close to the base integration. For the examples shown in this paper (substrate thickness $t<0.0001 \lambda$ ), as many as 240 points were needed in the surface integration of the metallic printed areas in order to treat conveniently this quasi-singular situation. When the thickness of the substrate increases up to $(t>0.01 \lambda)$, then only 24 points are needed to achieve a similar degree of accuracy.

[15] To overcome this problem, a different numerical treatment, based on the ideas reported in the work of $L u$ and Chew [2000] and $L u$ and $Y u$ [2002] has been implemented. In this case a full Galerkin approach employing rooftop functions inside the dielectric objects is formulated (see Figure 4 for the definition of the basis and testing functions). The first advantage of this technique is that it produces a fully symmetrical matrix system. There are special numerical techniques for accelerating the inversion of complex symmetrical matrices [Anderson et al., 1999]. A gain of at least a factor of two has been observed when using this specially tailored numerical techniques.

[16] Another important aspect of this technique is how to terminate properly the mesh of the dielectric objects. As it can be seen in equation (3), the polarization current is proportional to the total electric field. Since the electric field is not zero at the outer surface, then the polarization current is also not zero at this surface when looking form inside the dielectric object. In order to respect this behavior, the volume mesh must be truncated using half-rooftop functions. In addition, the polarization current is zero just outside the volume of the dielectric. This is why the added half-rooftop functions must be discontinuous, as shown in Figure 5. This discontinuous half-rooftop functions take into account for the discontinuity of the polarization currents, which are zero outside the dielectric but different from zero at the external surface. In addition, for all the interactions the gradient of the electric scalar potential is transferred to the testing function. To perform this transferring, the divergence theorem is applied:

$$
\begin{aligned}
\left\langle\nabla \Phi, \bar{f}_{m}^{d}(\bar{r})\right\rangle= & \int_{S_{m d}} \Phi \bar{f}_{m}^{d}(\bar{r}) \cdot \hat{e}_{n} d S_{d} \\
& -\int_{V_{m d}} \Phi \nabla \cdot \bar{f}_{m}^{d}(\bar{r}) d V_{d}
\end{aligned}
$$

where $\hat{e}_{n}$ is the outward unitary vector normal to the surface enclosing the testing function $\left(S_{m d}\right)$.

[17] In this equation, the first integral is always zero, since all rooftop testing functions are defined such that: $\bar{f}_{m}^{d}(\bar{r}) \cdot \hat{e}_{n}=0$. However, the discontinuity of the halfrooftop functions used to terminate the mesh, splits the second integral of (13) into an additional surface integral extended to the outer surface enclosing the volume of the dielectric object $\left(\Omega_{p}\right)$. The divergence of this function, then, leads to two different contributions. The linear term is transformed into a volume integral, while the discontinuity produces an additional integral extended to the external surface (see Figure 5).

[18] Taking into consideration all these aspects, the process of transferring the gradient from the Green's functions to the testing function leads to the following three terms for the case of the interaction between an homogeneous dielectric object and a metallic area:

$$
\begin{aligned}
Z_{m, n}^{(d, e)}= & Z_{n, m}^{(e, d)}=j \omega \int_{V_{p}} \bar{f}_{m}^{d}(\bar{r}) d V \int_{S_{e}} \bar{f}_{n}^{e}\left(\bar{r}^{\prime}\right) \overline{\bar{G}}_{A}\left(\bar{r}, \bar{r}^{\prime}\right) d S^{\prime} \\
& -\frac{1}{j \omega} \int_{\Omega_{p}} \hat{e}_{m} \cdot \bar{f}_{m}^{d}(\bar{r}) d S \int_{S_{e}} \nabla^{\prime} \cdot \bar{f}_{n}^{e}\left(\bar{r}^{\prime}\right) G_{V}\left(\bar{r}, \bar{r}^{\prime}\right) \\
& \cdot d S^{\prime}+\frac{1}{j \omega} \int_{V_{p}} \nabla \cdot \bar{f}_{m}^{d}(\bar{r}) d V \int_{S_{e}} \nabla^{\prime} \cdot \bar{f}_{n}^{e}\left(\bar{r}^{\prime}\right) \\
& \cdot G_{V}\left(\bar{r}, \bar{r}^{\prime}\right) d S^{\prime}
\end{aligned}
$$




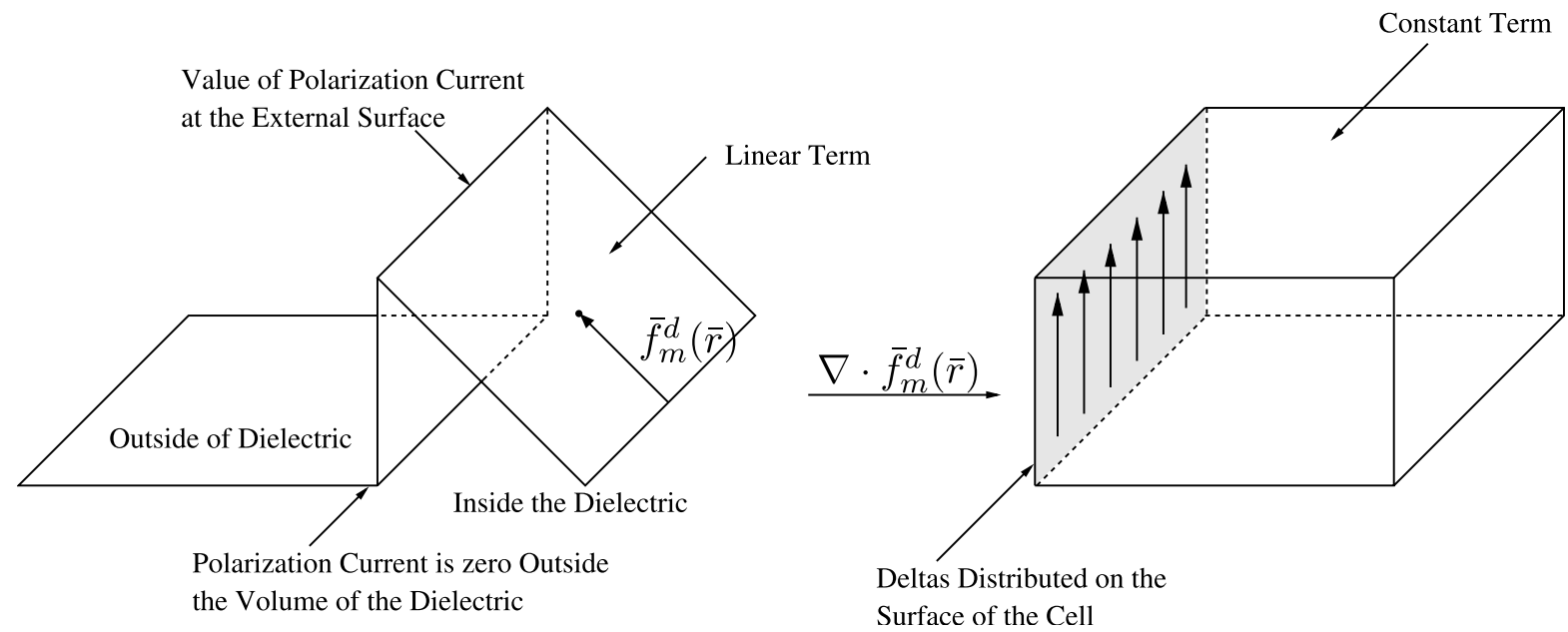

Figure 5. Half-rooftop functions used to terminate the mesh for the polarization currents.

where $\Omega_{p}$ is again the surface enclosing the volume of the dielectric object, and $\hat{e}_{m}$ is the outward unitary vector normal to this surface at the observation points. If the dielectric is inhomogeneous, a formulation where the integral equation unknown is the electric flux density $\bar{D}(\bar{r})$ is preferred [Schaubert et al., 1984]. For the case of homogeneous dielectric both formulations are equivalent.

[19] Using these basis and testing functions, the dielectric-dielectric interactions involve the computation of six order integrals, as shown in (15):

$$
\begin{aligned}
Z_{m, n}^{(d, d)}= & \frac{\int_{V_{p}} \bar{f}_{m}^{d}(\bar{r}) \cdot \bar{f}_{n}^{d}(\bar{r}) d V}{j \omega\left(\varepsilon-\varepsilon_{0}\right)}+j \omega \int_{V_{p}} \bar{f}_{m}^{d}(\bar{r}) d V \int_{V_{p}} \bar{f}_{n}^{d}\left(\bar{r}^{\prime}\right) \\
& \cdot \overline{\bar{G}}_{A}\left(\bar{r}, \bar{r}^{\prime}\right) d V^{\prime}-\frac{1}{j \omega} \int_{\Omega_{p}} \hat{e}_{m} \cdot \bar{f}_{m}^{d}(\bar{r}) d S \int_{V_{p}} \nabla^{\prime} \cdot \bar{f}_{n}^{d} \\
& \cdot\left(\bar{r}^{\prime}\right) G_{V}\left(\bar{r}, \bar{r}^{\prime}\right) d V^{\prime}+\frac{1}{j \omega} \int_{V_{p}} \nabla \cdot \bar{f}_{m}^{d}(\bar{r}) d V \\
& \cdot \int_{V_{p}} \nabla^{\prime} \cdot \bar{f}_{n}^{d}\left(\bar{r}^{\prime}\right) G_{V}\left(\bar{r}, \bar{r}^{\prime}\right) d V^{\prime}-\frac{1}{j \omega} \int_{V_{p}} \nabla \cdot \bar{f}_{m}^{d}(\bar{r}) \\
& \cdot d V \int_{\Omega_{p}} \hat{e}_{n} \cdot \bar{f}_{n}^{d}\left(\bar{r}^{\prime}\right) G_{V}\left(\bar{r}, \bar{r}^{\prime}\right) d S^{\prime}+\frac{1}{j \omega} \\
& \cdot \int_{\Omega_{p}} \hat{e}_{m} \cdot \bar{f}_{m}^{d}(\bar{r}) d S \int_{\Omega_{p}} \hat{e}_{n} \cdot \bar{f}_{n}^{d}\left(\bar{r}^{\prime}\right) G_{V}\left(\bar{r}, \bar{r}^{\prime}\right) d S^{\prime}
\end{aligned}
$$

From this last expression we can observe another set of surface integrals. They correspond again to the discontinuity of the polarization currents at the half-rooftop basis functions used to terminate the mesh at the dielectric external surface.

[20] From above equation, we can conclude that the evaluation of all the reaction integrals requires the treatment of weak singularities of the type $(1 / r)$. In the present work these weak singularities are treated numerically following simple procedures. The singular surface integrals are evaluated using polar coordinates, since the Jacobean of the polar transformation exactly compensate the weak singularity of the Green's functions. Following the same idea, the volume singular integrals are evaluated using spherical coordinates. In this case the Jacobean of the transformation is $\left(r^{2}\right)$, which completely annihilate the weak $(1 / r)$ singularity of the Green's functions. The total function to be integrated behaves very smoothly, thus requiring only 64 points for each volume integral to achieve convergence, even for very small substrate thickness. All other nonsingular integrals are treated with special cubature formulas derived in brick domains [Cools, 1999]. Using these cubature rules, a volume integral is reduced to a one-dimensional summation, involving the function evaluated in $(N)$ strategic points, with associated weights:

$$
I=\int_{V_{p}} f(\bar{r}) d V=\sum_{i=1}^{N} w_{i} f\left(\bar{r}_{i}\right)
$$

[21] For the integration in spherical coordinates, the volume of the brick cell is decomposed in four pyramids, following the strategy sketched in Figure 6. After some cumbersome but straightforward manipulations, the original integral is decomposed in several integrals of the following type (note that the singularity of the Green's functions is effectively extracted):

$$
\begin{aligned}
I_{1}= & \int_{\phi_{1}=\arctan _{2} \frac{y_{1}-y_{m}}{x_{2}-x_{m}}}^{\phi_{2}=\arctan _{2} \frac{y_{2}-y_{m}}{x_{m}-x_{m}}} \int_{\theta_{1}=\arctan _{2} \frac{x_{2}-x_{m}}{\left(z_{2}-z_{m}\right) \cos \phi}}^{\theta_{2}=\arctan _{2} \frac{x_{2}-x_{m}}{\left(z_{1}-z_{m}\right) \cos \phi}} \\
& \cdot \int_{r_{1}=0}^{r_{2}=\frac{x_{2}-x_{m}}{\sin \theta \cos \phi}} \frac{e^{-j k_{0} r}}{r} r^{2} \sin \theta d r d \theta d \phi
\end{aligned}
$$




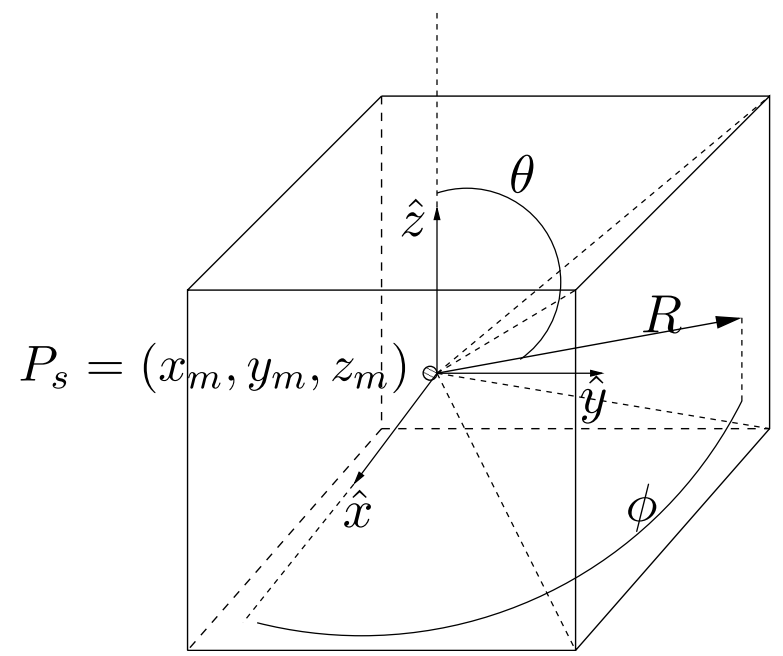

Figure 6. Geometry for the singularity extraction in a brick cell.

where $x=x_{2}, y=y_{2}, x=x_{1}, y=y_{1}, z=z_{1}$ and $z=z_{2}$ define the planes where the six cube sides are placed, and all other parameters are shown in Figure 6. Above expression contains the integration of a very smooth function, and numerical integration converges fast.

[22] Another interesting aspect related to the choice of the basis function is the modeling of the equivalent surface charge induced on the outer surfaces of the dielectric bodies. If pulse basis functions are selected, then the charge induced on the outer surfaces can correctly be modeled without any further treatment. In this case the surface integrals on the opposite sides of each pulse cell gives the desired charge as shown in Figure 3. The two opposite sides of contiguous cells tend to cancel out the corresponding charge inside the dielectric body Figure 3. Only the sides describing the external surface do not have a contiguous cell, therefore leading to the total net charge induced on the outer surface of dielectric bodies. However, if rooftop basis functions are defined on brick cells, then a zero normal component of the electric field vector $(\bar{E})$ is imposed on the outer surfaces of dielectric bodies. This goes clearly against the boundary condition for the normal component of the electric field at a dielectric interface. As already mentioned, to avoid this problem half-rooftop functions need to be defined in order to terminate correctly the meshes of the dielectric bodies. They are also used to properly model the equivalent charge induced on the associated surfaces. The presence of these half-rooftop functions assures the correct modeling of the charge, at the air-dielectric interface, through the second integral of equation (14).

\section{Polarization Currents Versus Electric Flux Density}

[23] In equation (8), the polarization current is the unknown of the integral equation, and it is expanded in terms of volumetric pulse or rooftop basis functions. An alternative formulation can be foreseen if we take the electric flux density vector as unknown of the integral equation [Schaubert et al., 1984]. It is essential to keep in mind that both homogeneous and nonhomogeneous dielectrics can be formulated with both, the electric flux density $(\bar{D})$ or the polarization currents $\left(\bar{J}_{p}\right)$, as unknowns. While for nonhomogeneous dielectrics it is simpler to formulate the integral equation with $(\bar{D})$, for homogeneous dielectrics it is the opposite. The complexity of the formulations lies in the possibility of formulating fully symmetrical MoM matrices. For homogeneous dielectrics, the polarization currents formulation leads to fully symmetrical matrices with a straightforward MoM implementation. If the $(\bar{D})$ formulation is employed, then a redefinition of the testing functions must be employed if fully symmetrical matrices are desired.

[24] If the dielectric is not homogeneous, there exist also two possibilities: The first and most commonly used choice is to redefine the problem in terms of the electric flux density $(\bar{D})$, which is continuous across the medium changes. The second possibility is to expand the polarization currents $\left(\bar{J}_{p}\right)$ in terms of a new set of discontinuous rooftop basis functions, which take into account for the medium variation (discontinuity of $\bar{J}_{s}$ ) between adjacent cells. This formulation is less popular for obvious reasons, namely it adds complexity with respect the classical $(\bar{D})$ formulation, where the unknown is always continuous.

[25] For the case of homogeneous dielectrics, both formulations are equivalent, and lead to algorithms of similar complexity. To show that this is indeed the case, we first express the polarization current $\left(\bar{J}_{p}\right)$ in terms of the total electric field inside the dielectric, as in (3):

$$
\bar{J}_{p}\left(\bar{r}^{\prime}\right)=j \omega\left[\hat{\epsilon}\left(\bar{r}^{\prime}\right)-\varepsilon_{0}\right] \bar{E}\left(\bar{r}^{\prime}\right)
$$

where now we have used the dielectric permittivity distribution inside the material body $(\hat{\epsilon})$. Following the same steps as in the work of Schaubert et al. [1984], the medium constant ratio $(\kappa)$ is defined as:

$$
\kappa\left(\bar{r}^{\prime}\right)=\frac{\hat{\epsilon}\left(\bar{r}^{\prime}\right)-\varepsilon_{0}}{\hat{\epsilon}\left(\bar{r}^{\prime}\right)}
$$


The relation between the electric flux density $(\bar{D})$ and the electric field is given by:

$$
\bar{D}\left(\bar{r}^{\prime}\right)=\hat{\epsilon}\left(\bar{r}^{\prime}\right) \bar{E}\left(\bar{r}^{\prime}\right)
$$

Using the new definitions, the polarization current $\left(\bar{J}_{p}\right)$ can then be written as follows:

$$
\bar{J}_{p}\left(\bar{r}^{\prime}\right)=j \omega \kappa\left(\bar{r}^{\prime}\right) \bar{D}\left(\bar{r}^{\prime}\right)
$$

Now, if we employ $(\bar{D})$ as the unknown for the dielectric regions, then the dielectric-dielectric interactions can be expressed as follows:

$$
\begin{aligned}
Z_{m, n}^{(d, d)}= & \frac{\int_{V_{p}} \bar{f}_{m}^{d}(\bar{r}) \cdot \bar{f}_{n}^{d}(\bar{r}) d V}{\hat{\epsilon}(\bar{r})}-\omega^{2} \int_{V_{p}} \bar{f}_{m}^{d}(\bar{r}) d V \\
& \cdot \int_{V_{p}} \kappa\left(\bar{r}^{\prime}\right) \bar{f}_{n}^{d}\left(\bar{r}^{\prime}\right) \overline{\bar{G}}_{A}\left(\bar{r}, \bar{r}^{\prime}\right) d V^{\prime}-\int_{V_{p}} \bar{f}_{m}^{d}(\bar{r}) d V \\
& \cdot \nabla \int_{V_{p}} \nabla^{\prime} \cdot\left[\kappa\left(\bar{r}^{\prime}\right) \bar{f}_{n}^{d}\left(\bar{r}^{\prime}\right)\right] G_{V}\left(\bar{r}, \bar{r}^{\prime}\right) d V^{\prime}
\end{aligned}
$$

If the divergence theorem given in (13), and the following nabla operator product rule are applied:

$$
\begin{aligned}
\nabla^{\prime} \cdot\left[\kappa\left(\bar{r}^{\prime}\right) \bar{f}_{n}^{d}\left(\bar{r}^{\prime}\right)\right]= & \nabla^{\prime} \kappa\left(\bar{r}^{\prime}\right) \cdot \bar{f}_{n}^{d}\left(\bar{r}^{\prime}\right) \\
& +\kappa\left(\bar{r}^{\prime}\right) \nabla^{\prime} \cdot \bar{f}_{n}^{d}\left(\bar{r}^{\prime}\right)
\end{aligned}
$$

then, equation (22) can be further transformed into:

$$
\begin{aligned}
Z_{m, n}^{(d, d)}= & \frac{\int_{V_{p}} \bar{f}_{m}^{d}(\bar{r}) \cdot \bar{f}_{n}^{d}(\bar{r}) d V}{\hat{\epsilon}(\bar{r})}-\omega^{2} \int_{V_{p}} \bar{f}_{m}^{d}(\bar{r}) d V \\
& \cdot \int_{V_{p}} \kappa\left(\bar{r}^{\prime}\right) \bar{f}_{n}^{d}\left(\bar{r}^{\prime}\right) \overline{\bar{G}}_{A}\left(\bar{r}, \bar{r}^{\prime}\right) d V^{\prime} \\
& -\int_{\Omega_{p}} \hat{e}_{m} \cdot \bar{f}_{m}^{d}(\bar{r}) d S \int_{V_{p}} \kappa\left(\bar{r}^{\prime}\right) \nabla^{\prime} \bar{f}_{n}^{d}\left(\bar{r}^{\prime}\right) \\
& \cdot G_{V}\left(\bar{r}, \bar{r}^{\prime}\right) d V^{\prime}+\int_{V_{p}} \nabla \cdot \bar{f}_{m}^{d}(\bar{r}) d V \int_{V_{p}} \kappa\left(\bar{r}^{\prime}\right) \nabla^{\prime} \\
& \cdot \bar{f}_{n}^{d}\left(\bar{r}^{\prime}\right) G_{V}\left(\bar{r}, \bar{r}^{\prime}\right) d V^{\prime}+\int_{V_{p}} \nabla \cdot \bar{f}_{m}^{d}(\bar{r}) d V \\
& \cdot \int_{V_{p}} \nabla^{\prime} \kappa\left(\bar{r}^{\prime}\right) \bar{f}_{n}^{d}\left(\bar{r}^{\prime}\right) G_{V}\left(\bar{r}, \bar{r}^{\prime}\right) d V^{\prime} \\
& -\int_{\Omega_{p}} \hat{e}_{m} \cdot \bar{f}_{m}^{d}(\bar{r}) d S \int_{V_{p}} \nabla^{\prime} \kappa\left(\bar{r}^{\prime}\right) \cdot \bar{f}_{n}^{d}\left(\bar{r}^{\prime}\right) \\
& \cdot G_{V}\left(\bar{r}, \bar{r}^{\prime}\right) d V^{\prime}
\end{aligned}
$$

The term $\nabla^{\prime} \kappa\left(\bar{r}^{\prime}\right)$ is null if the medium does not change between adjacent cells $\left(\kappa\left(\bar{r}^{\prime}\right)=\right.$ constant $)$. However, if there exist a medium change, then the volume integrals where the term $\nabla^{\prime} \kappa\left(\bar{r}^{\prime}\right)$ appears, are transformed to surface integrals defined on the interface between nonhomogeneous cells.

[26] If the dielectric is homogeneous, the last two terms are null except for the half-rooftop basis functions defined on the external surface. In this case, the dielectricdielectric interactions can be evaluated as follows:

$$
\begin{aligned}
Z_{m, n}^{(d, d)}= & \frac{\int_{V_{p}} \bar{f}_{m}^{d}(\bar{r}) \cdot \bar{f}_{n}^{d}(\bar{r}) d V}{\hat{\epsilon}(\bar{r})}-\omega^{2} \int_{V_{p}} \bar{f}_{m}^{d}(\bar{r}) d V \\
& \cdot \int_{V_{p}} \kappa\left(\bar{r}^{\prime}\right) \bar{f}_{n}^{d}\left(\bar{r}^{\prime}\right) \overline{\bar{G}}_{A}\left(\bar{r}, \bar{r}^{\prime}\right) d V^{\prime} \\
& -\int_{\Omega_{p}} \hat{e}_{m} \cdot \bar{f}_{m}^{d}(\bar{r}) d S \int_{V_{p}} \kappa\left(\bar{r}^{\prime}\right) \nabla^{\prime} \bar{f}_{n}^{d}\left(\bar{r}^{\prime}\right) \\
& \cdot G_{V}\left(\bar{r}, \bar{r}^{\prime}\right) d V^{\prime}+\int_{V_{p}} \nabla \cdot \bar{f}_{m}^{d}(\bar{r}) d V \int_{V_{p}} \kappa\left(\bar{r}^{\prime}\right) \nabla^{\prime} \\
& \cdot \bar{f}_{n}^{d}\left(\bar{r}^{\prime}\right) G_{V}\left(\bar{r}, \bar{r}^{\prime}\right) d V^{\prime}-\int_{V_{p}} \nabla \cdot \bar{f}_{m}^{d}(\bar{r}) d V \\
& \cdot \int_{\Omega_{p}} \kappa\left(\bar{r}^{\prime}\right) \hat{e}_{n} \cdot \bar{f}_{n}^{d}\left(\bar{r}^{\prime}\right) G_{V}\left(\bar{r}^{\prime}, \bar{r}^{\prime}\right) d S^{\prime} \\
& +\int_{\Omega_{p}} \hat{e}_{m} \cdot \bar{f}_{m}^{d}(\bar{r}) d S \int_{\Omega_{p}} \kappa\left(\bar{r}^{\prime}\right) \hat{e}_{n} \cdot \bar{f}_{n}^{d}\left(\bar{r}^{\prime}\right) \\
& \cdot G_{V}\left(\bar{r}, \bar{r}^{\prime}\right) d S^{\prime}
\end{aligned}
$$

where $\left(\Omega_{p}\right)$ is, as before, the dielectric body external surface, and $\left(\hat{e}_{m}, \hat{e}_{n}\right)$ are the unit vectors normal to observation and source brick faces, where the basis and test functions are defined, respectively.

[27] The last expression is very similar to equation (15). The only differences are in the multiplying constants. Moreover, If the electric flux density $(\vec{D})$ is chosen as the expansion unknown, and the relations (13) and (23) are taken into account, then the cross interactions between conducting and dielectric cells can be written as:

$$
\begin{aligned}
Z_{m, n}^{(d, e)}= & j \omega \int_{V_{p}} \bar{f}_{m}^{d}(\bar{r}) d V \int_{S_{e}} \bar{f}_{n}^{e}\left(\bar{r}^{\prime}\right) \overline{\bar{G}}_{A}\left(\bar{r}, \bar{r}^{\prime}\right) d S^{\prime} \\
& -\frac{1}{j \omega} \int_{\Omega_{p}} \hat{e}_{m} \cdot \bar{f}_{m}^{d}(\bar{r}) d S \int_{S_{e}} \nabla^{\prime} \cdot \bar{f}_{n}^{e}\left(\bar{r}^{\prime}\right) \\
& \cdot G_{V}\left(\bar{r}, \bar{r}^{\prime}\right) d S^{\prime}+\frac{1}{j \omega} \int_{V_{p}} \nabla \cdot \bar{f}_{m}^{d}(\bar{r}) d V \\
& \cdot \int_{S_{e}} \nabla^{\prime} \cdot \bar{f}_{n}^{e}\left(\bar{r}^{\prime}\right) G_{V}\left(\bar{r}, \bar{r}^{\prime}\right) d S^{\prime}
\end{aligned}
$$


for the case when the source is on the conductor and the observation inside the dielectric, or it can be written as:

$$
\begin{aligned}
Z_{m, n}^{(e, d)}= & -\omega^{2} \int_{S_{e}} \bar{f}_{m}^{e}(\bar{r}) d S \int_{V_{p}} \kappa\left(\bar{r}^{\prime}\right) \bar{f}_{n}^{d}\left(\bar{r}^{\prime}\right) \overline{\bar{G}}_{A}\left(\bar{r}, \bar{r}^{\prime}\right) d V^{\prime} \\
& +\int_{S_{e}} \nabla \cdot \bar{f}_{m}^{e}(\bar{r}) d S \int_{V_{p}} \nabla^{\prime} \kappa\left(\bar{r}^{\prime}\right) \cdot \bar{f}_{n}^{d}\left(\bar{r}^{\prime}\right) \\
& \cdot G_{V}\left(\bar{r}, \bar{r}^{\prime}\right) d V^{\prime}+\int_{S_{e}} \nabla \cdot \bar{f}_{m}^{e}(\bar{r}) d S \\
& \cdot \int_{V_{p}} \kappa\left(\bar{r}^{\prime}\right) \nabla^{\prime} \cdot \bar{f}_{n}^{d}\left(\bar{r}^{\prime}\right) G_{V}\left(\bar{r}, \bar{r}^{\prime}\right) d V^{\prime}
\end{aligned}
$$

if, otherwise, the source is inside the dielectric and the observation on the conductor. Furthermore, if the medium is homogeneous, (27) can be written as:

$$
\begin{aligned}
Z_{m, n}^{(e, d)}= & -\omega^{2} \int_{S_{e}} \bar{f}_{m}^{e}(\bar{r}) d S \int_{V_{p}} \kappa\left(\bar{r}^{\prime}\right) \bar{f}_{n}^{d}\left(\bar{r}^{\prime}\right) \overline{\bar{G}}_{A}\left(\bar{r}, \bar{r}^{\prime}\right) d V^{\prime} \\
& -\int_{S_{e}} \nabla \cdot \bar{f}_{m}^{e}(\bar{r}) d S \int_{\Omega_{p}} \kappa\left(\bar{r}^{\prime}\right) \hat{e}_{n} \cdot \bar{f}_{n}^{d}\left(\bar{r}^{\prime}\right) \\
& \cdot G_{V}\left(\bar{r}, \bar{r}^{\prime}\right) d V^{\prime}+\int_{S_{e}} \nabla \cdot \bar{f}_{m}^{e}(\bar{r}) d S \\
& \cdot \int_{V_{p}} \kappa\left(\bar{r}^{\prime}\right) \nabla^{\prime} \cdot \bar{f}_{n}^{d}\left(\bar{r}^{\prime}\right) G_{V}\left(\bar{r}, \bar{r}^{\prime}\right) d V^{\prime}
\end{aligned}
$$

As it can be seen, if the constants are properly adjusted, this expression is reciprocal to (26). Moreover, the last expressions (26) and (28) are equivalent for homogeneous dielectric to equation (14), showing that in this case the problem can be formulated indistinctly by using as unknown the electric flux density $(\bar{D})$ or the volumetric polarization current $\left(\vec{J}_{p}\right)$.

\section{Excitation Model}

[28] For the excitation of microstrip circuits and printed antennas, we have used the simple $\delta$ gap model described in the work of Eleftheriades and Mosig, 1996]. In this model, a half-rooftop function is added at the beginning of the input feeding line, so that a voltage simulating the effect of the signal generator can be applied at this point. This half-rooftop function also takes into account for the current flowing from the generator to the circuit. In traditional models, the voltage applied as excitation has as reference point the infinite ground plane of the microstrip circuit. This infinite ground plane reference for the excitation voltage is automatically accounted for with the use of the multilayered media Green's functions. It is the Green's functions, therefore, which automatically add the reference for the applied voltage.

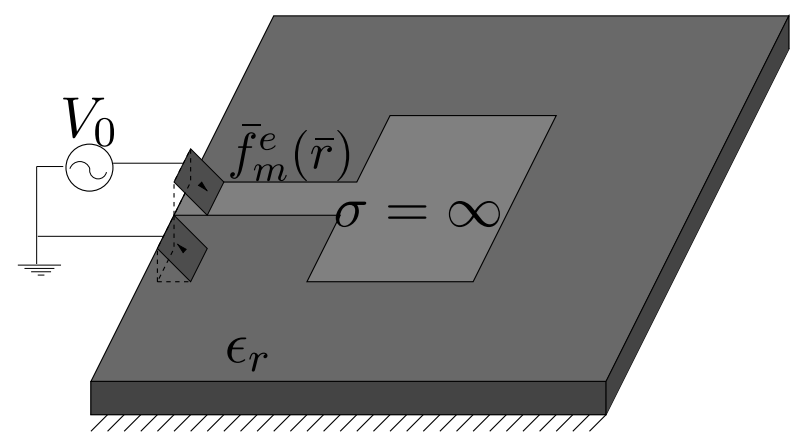

Figure 7. Microstrip antenna excitation model.

[29] In the integral equation formulated in this paper for finite size dielectric substrates, however, the Green's functions do not take into consideration any ground plane reference. The model, then, must be modified to properly include a valid reference point for the voltage applied to the input feeding line. In this work we have used the basic excitation model presented in the work of Tiezzi et al., 2000b] for finite size ground planes.

[30] In this modified model, a second half-rooftop function is defined in the ground plane, at the point where the excitation is placed. This second half-rooftop, together with the half-rooftop defined on the input feeding line, form a new split full basis function Figure 7. The basis function composed by these two half-rooftop functions take into account for the current flowing from the ground plane to the input feeding line, through the voltage generator. The method can be easily generalized if more than two coupled half-rooftop functions are to be used.

[31] On the basis of these basic ideas, the model presented in the work of Tiezzi et al. [2000b] for finite size ground planes has been extended, so that asymmetric cell sizes can be used on the input feeding line and on the ground plane. In the original model proposed in the work of Tiezzi et al. [2000b], the half-rooftop functions defined on the input feeding line and on the ground plane must be of the same size. This is because they formulated the excitation vector of the system using directly the current density induced on the new full basis function formed with the two split half-rooftop functions. Since the total current flowing on the input feeding line and in the ground plane (through the voltage generator) must be the same, then the cell sizes must also be the same.

[32] In many applications however, it would be useful to mesh the ground plane using different cell sizes than the input feeding line. This is the case, for instance, when using triangular cells unstructured meshes, or when you want to reduce the number of total unknowns used in the matrix system to represent the circuit. This last issue is of vital importance in the IE derived in this paper for finite 


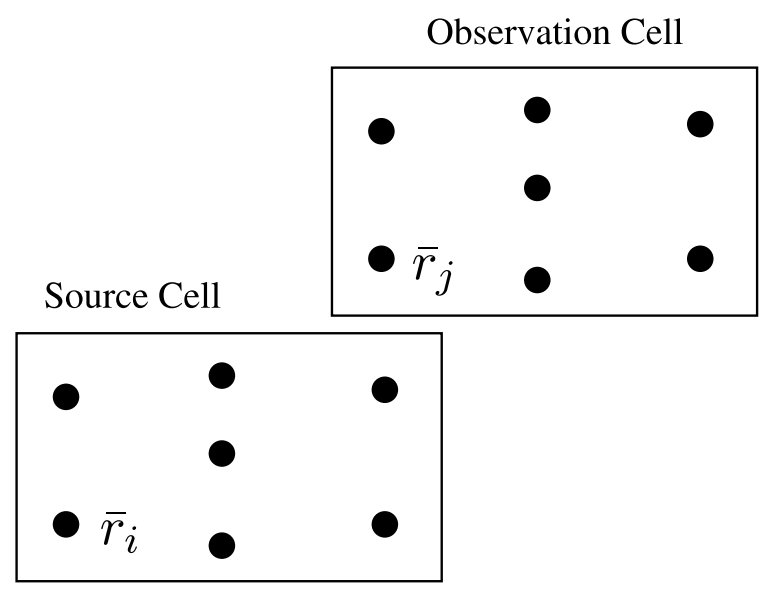

(a) Situation with no partial overlapping.

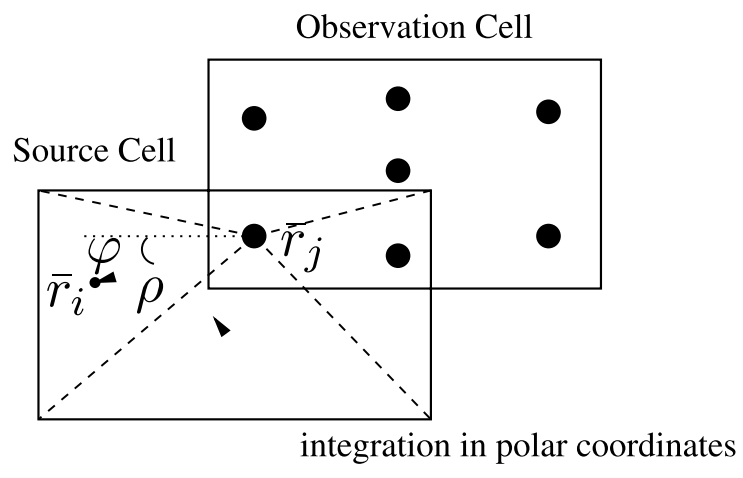

(b) Situation with partial overlapping.

Figure 8. Sketch of numerical challenges associated to the singularity extraction in partial overlapping situations. Situation with no partial overlapping.

size dielectric substrates. This is because the discretization of the dielectric substrates tends to increase considerably the size of the global matrix system, and techniques must be derived in order to give enough flexibility to the geometrical discretization to keep this size to a minimum. The modified version of the excitation model is one of such techniques, since it allows to discretize the ground plane with a number of cells independent from the width of the input feeding line. The second technique derived in this paper for this purpose is the Meshing Overlapping strategy between dielectric objects and touching metallic areas to be presented in section 6 .

[33] The key point to extend the excitation model presented in the work of Tiezzi et al. [2000b], so that different cell sizes can be used in the definition of the two half-rooftop functions, is to represent the excitation vector of the system with the total current flowing on the new basis function excitation. This is easily accomplished by normalizing the excitation vector with the widths of the two cells in which the excitation is defined. The current density on each half-rooftop used in the definition of the excitation can, in general, be different $\left(\bar{J}_{1}, \bar{J}_{2}\right)$. However, the total current flowing through the basis function must be unique, so that the following restriction is imposed: $\bar{J}_{1} w_{1}=\bar{J}_{2} w_{2}$, where $w_{1}$ is the width of the first half-rooftop and $w_{2}$ is the width of the second half-rooftop.

[34] Following this procedure, the unknown coefficients of the matrix system represent the current density induced on the metallic areas, except at the point of excitation, where they represent the total current flowing at this point from the voltage generator. In this way the continuity of the current is assured between the ground plane and the feeding line through the voltage generator, independently on the size of the two cells in which the half-rooftop functions are defined.

\section{Independent Meshing}

[35] One important issue concerning the IE method derived for the analysis of finite printed circuits and antennas, is the possibility of independently meshing the volume dielectric regions and the touching metallic surfaces. This is an important feature, since then different number of cells and different meshing strategies can be applied independently to dielectric regions and metallic areas in a given geometry. This clearly represents an advantage, since it increases the flexibility of the method to treat complex structures, and simplifies the geometrical treatment of the problem. Also it allows the use of uniform meshes in combination of the conjugate gradient-fast Fourier transform technique [Catedra et al., 1989].

[36] The formulation for the implementation of the independent meshing is the same as detailed in sections 2 and 3, and the MoM matrix is computed with the same expressions given in equations (7)-(11) for pulses, or equations (14), (15) for rooftop functions. On the contrary, the numerical treatment of the problem increases in complexity. If independent meshing is allowed, then partial singularities arising between surface charges induced on the dielectric and on the printed metallic areas must be conveniently treated. Consequently, a singularity extraction algorithm based on cells can not be performed as before. Instead, a new singularity extraction scheme based on test integration points must be applied. 


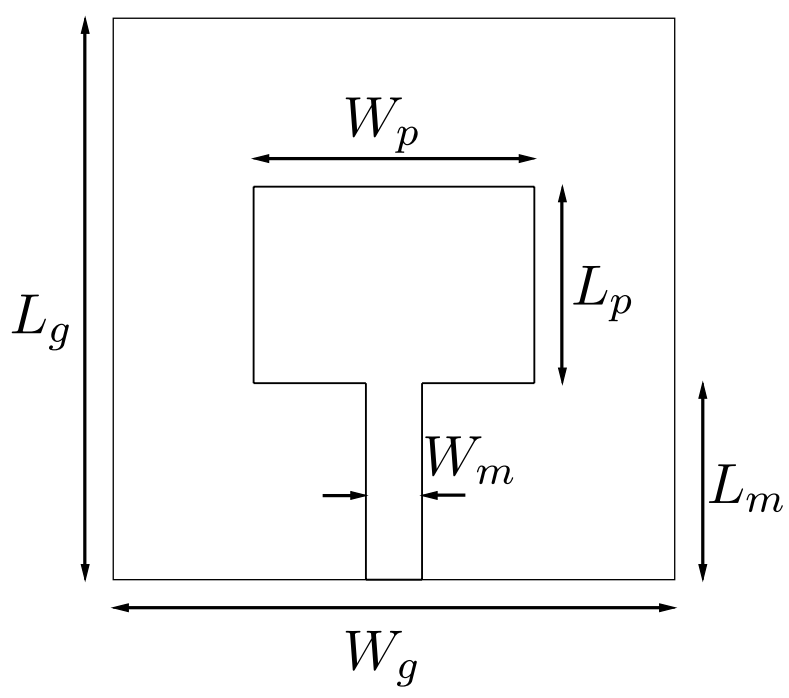

Figure 9. Printed patch antenna introduced in the work of Sarkar et al. [1990]. The dimensions are $L_{g}=15.0 \mathrm{~cm}$, $W_{g}=15.0 \mathrm{~cm}, L_{p}=5.0 \mathrm{~cm} W_{p}=5.0 \mathrm{~cm}, L_{m}=5.0 \mathrm{~cm}$, and $W_{m}=1.0 \mathrm{~cm}$. The dielectric permittivity is $\epsilon_{r}=2.56$, and the thickness $h=0.2 \mathrm{~cm}$.

[37] In this scheme, the singularity is extracted if a given integration point of the test function lies inside a given basis function. More precisely, for each observation point integration on the external surface of the dielectric, it is checked whether or not the point lies inside the current source cell belonging to a metallic area of the circuit (see Figure 8). If this is the case, then the integration for the current observation point extended to the corresponding source cell is performed in the polar plane, therefore effectively eliminating the singularity. If on the contrary, the observation point lies outside the current source cell, then a normal nonsingular integration procedure, as described in section 3 , is applied. The whole process is sketched in Figure 8a, which shows a situation where no partial overlapping occurs. In this case integration is always carried out using the cubature rules of Cools [1999]. On the contrary, Figure 8b shows a partial overlapping situation, where two integration points of the observation cell lays inside the source cell. For this two points, the integration extended to the source cell is carried out through transformation to the polar plane. For all other observer points, integration is again carried out using cubature rules.

[38] In this work the technique has been successfully implemented to allow for independent meshing of the dielectric regions and the printed metallic surfaces. It has been observed, however, that numerical convergence of the results is slower when large overlapping is allowed between dielectric regions and metallic areas. The slow convergence is associated to the boundary condition for the normal component of the electric flux density vector $(\bar{D})$. For a cell not touching a metallic area, the normal component of the electric flux density must be continuous through the external surface: $0=\hat{e}_{n} \cdot\left(\bar{D}_{2}-\bar{D}_{1}\right)$. However, if the cell is bellow a metallic area, then the normal component is related to the surface charge with the well known boundary condition: $\rho_{s}=\hat{e}_{n} \cdot\left(\bar{D}_{2}-\bar{D}_{1}\right)$. Finally, if there is a partial overlapping situation for a

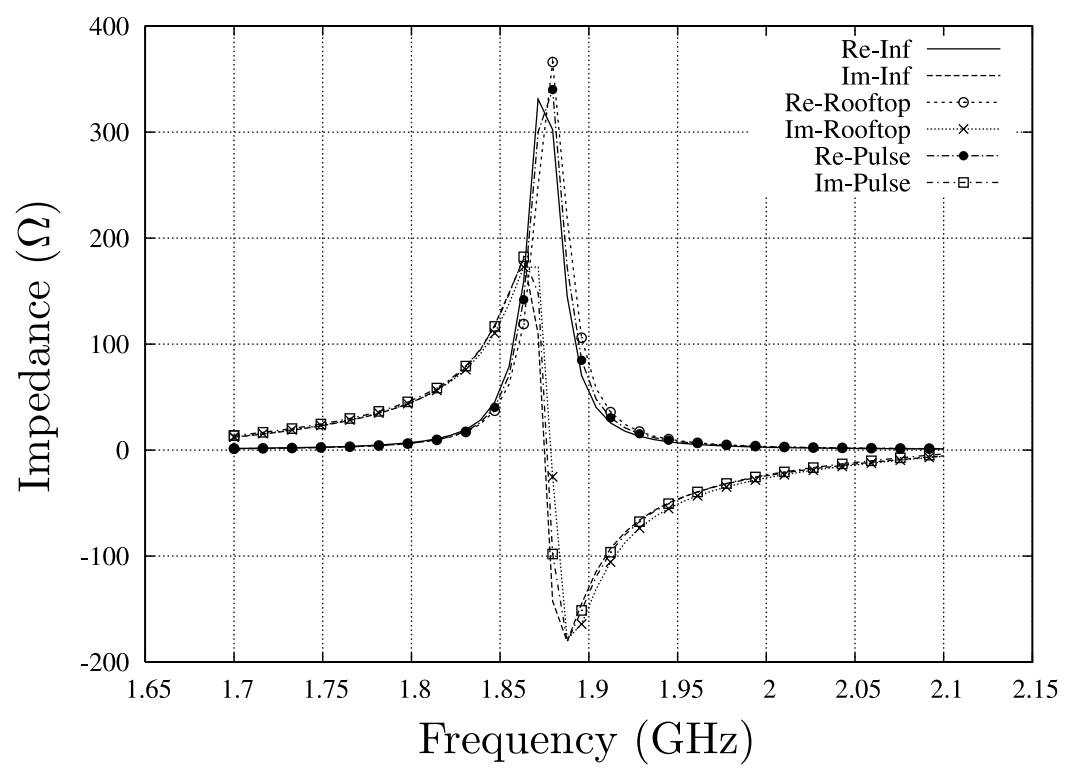

Figure 10. Input impedance of the antenna presented in the work of Sarkar et al. [1990]. 


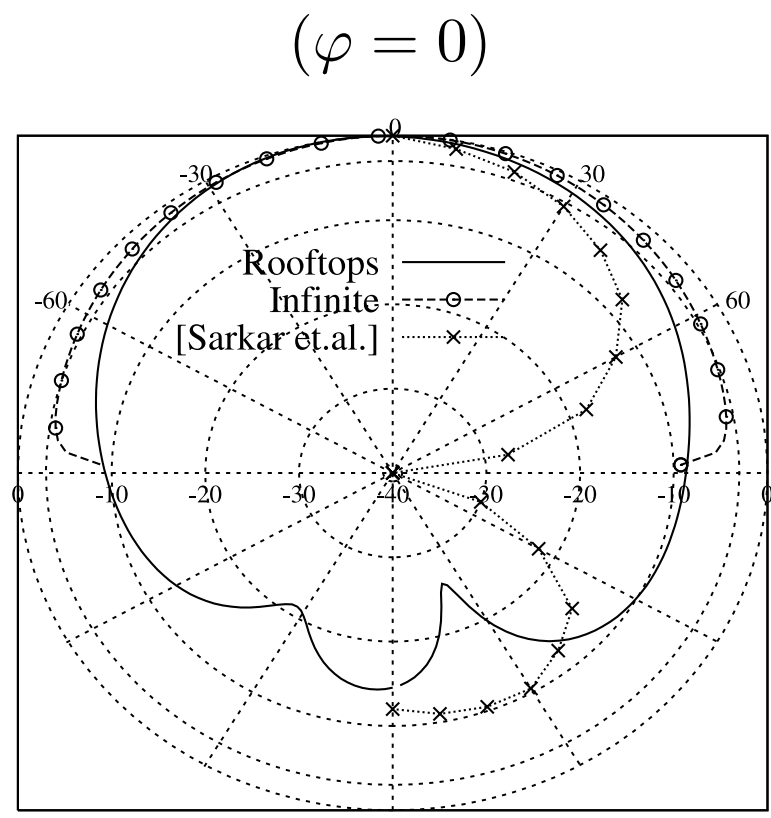

a) E-Plane (dB)
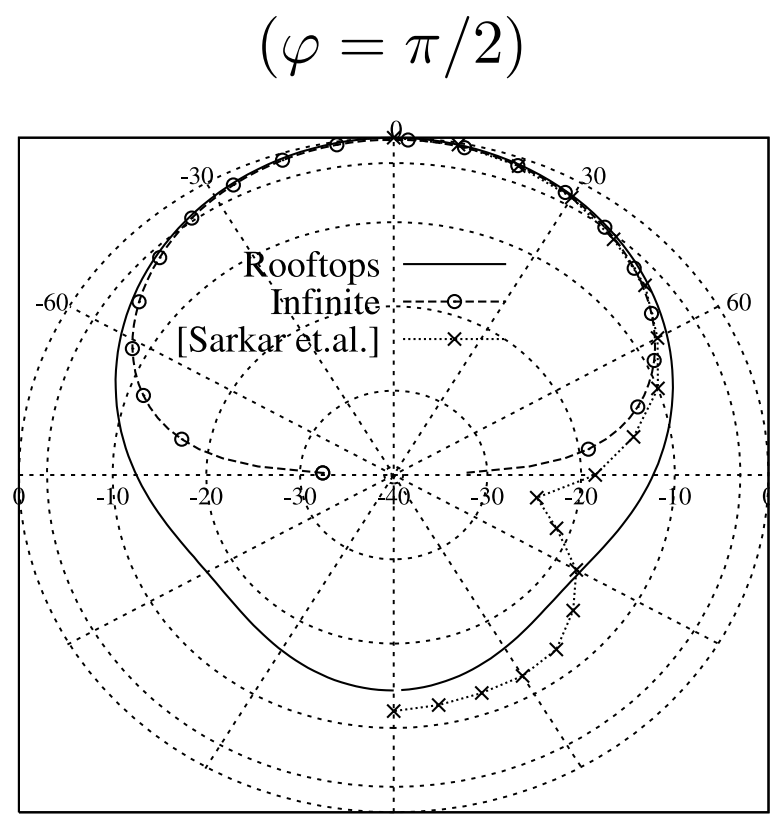

b) H-Plane (dB)

Figure 11. Radiation patterns of the antenna presented in the work of Sarkar et al. [1990].

given cell, then each condition must be imposed over the corresponding portion of the cell, touching or not the metal. This overlapped cell can not fulfill both conditions at the same time, so that an averaging process takes place. In section 7 , details on the convergence of the results with partial overlapping are included.

\section{Results}

[39] The efficient numerical implementation of the volume equivalent integral equation technique derived in this paper has allowed for the precise evaluation of the input impedance and radiation characteristics of finite microstrip antennas.

[40] The first structure investigated is the microstrip patch antenna presented in the work of Sarkar et al. [1990] (Figure 9). However, Sarkar et al. [1990] does not give results on the input impedance of the antenna, and in the work of Sarkar et al. [1990] the radiation patterns obtained with a volume equivalent and with a surface equivalent formulation do not agree well, therefore indicating the presence of inaccuracies. In Figure 10 we present for the first time the input impedance of this antenna. The figure shows the results obtained with the two approaches developed in this paper, and the results obtained with a spatial domain multilayered Green's functions infinite dielectric model [Mosig, 1988]. It can be seen that in this case the three techniques agree very well, and in particular a radiation resonance appears at $1.875 \mathrm{GHz}$. The radiation patterns of this structure at the resonant frequency are shown in Figure 11a (E plane) and Figure 11b (H plane). The figures only show the results obtained with rooftop in the substrate, since the results obtained with pulses are very similar. The figures also show the results obtained with the infinite substrate model, and the results presented in the work of Sarkar et al. [1990]. The radiation patterns are similar in all cases in the upper half-space, but not in the lower half-space. In particular, with the infinite substrate model there is no information about the radiation patterns in the backside region.

[41] To show the usefulness of the excitation method derived in section 7 , we have analyzed the same patch antenna shown in Figure 9, using different cell sizes in the definition of the excitation. Figure 12 shows the results obtained when the excitation is defined with cell widths of $10 \mathrm{~mm}$ in both the input line and in the ground plane, and compares them with results obtained when the cell size in the ground plane is changed to $5 \mathrm{~mm}$ and $15 \mathrm{~mm}$ (while maintaining constant the cell width in the input line). In spite of using different cell widths, the results in all cases are very similar, therefore demonstrating the usefulness of the approach derived. Furthermore, Figure 13 shows the relative deviation as a 


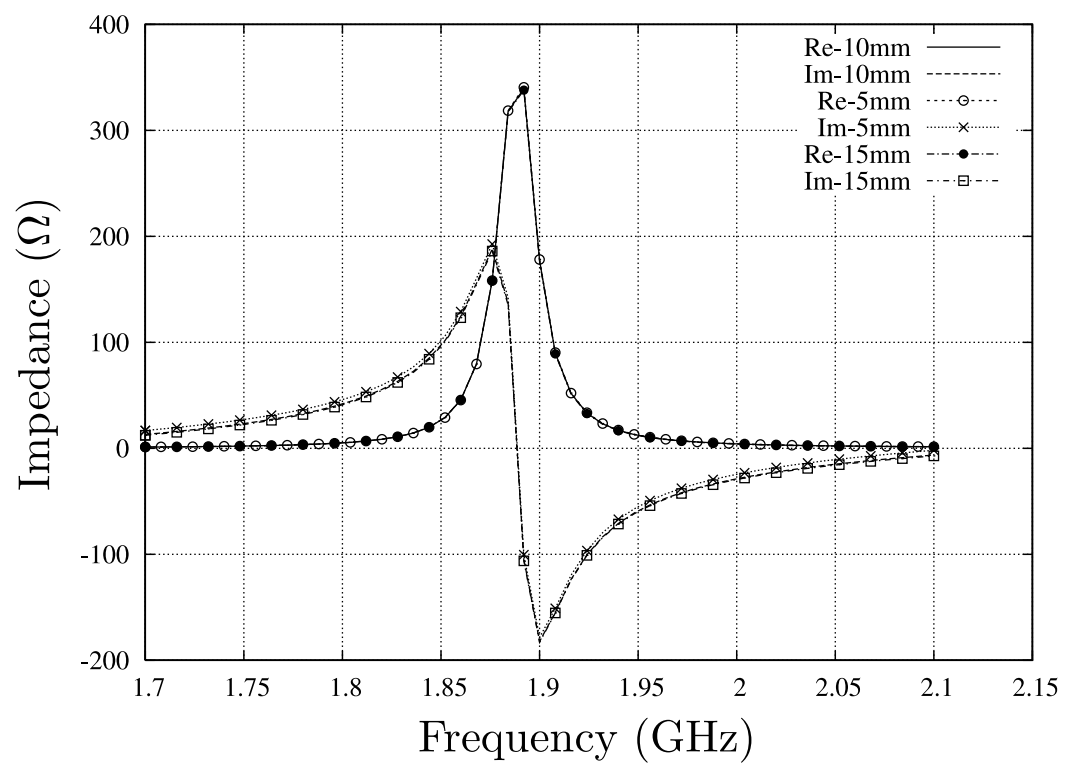

Figure 12. Input impedance of the patch antenna shown in Figure 9 using different cell sizes for the definition of the excitation.

function of frequency, for the $5 \mathrm{~mm}$ and $15 \mathrm{~mm}$ cases, taken the $10 \mathrm{~mm}$ width as the nominal situation (equal width for the cell in the input line and in the ground plane). It can be seen that from 1.7 to $1.95 \mathrm{GHz}$, the relative deviation remains always below $2 \%$.

[42] In order to verify the results obtained with the new numerical implementation of the volume equivalent integral equation derived in this paper, a microstrip patch antenna presented in the work of Tiezzi et al. [2000a] is studied (Figure 14). First, we present in Figure 15 the input impedance of this antenna, and we compare the results with data obtained from measurements. Also the results obtained with the infinite dielectric model are included for reference. It can be seen that in all cases the results are in good agreement, but the input impedance obtained with the new finite dielectric model is closer to measurements than the infinite dielectric model. In this case, then, it is important to take into account the side effects of the finite size dielectric substrate if accurate results are desired.

[43] Next, we present the radiation patterns of this structure at $5.94 \mathrm{GHz}$ in Figure 16a (E plane) and Figure 16b (H plane). The figures compare the results obtained with the new approach, with the infinite substrate results, and with measurements. It can be seen that the agreement is good, and in particular the backside radiation predicted by the new numerical model is very closed to the real measured radiation patterns.

[44] The numerical implementation of the integral equation derived in this paper has shown to be very robust, so that more complex finite-size multilayered structures can be analyzed. In Figure 17 we present a two layer microstrip patch antenna designed to operate with circular polarization. The antenna is fed with a standard microstrip line, which couples broadside to a patch placed on top. In the square patch a corner has been chop off as seen in Figure 17 for dual mode operation. The perturbation introduced by this cut corner couples the two degenerated radiating modes of the square patch. Moreover, the coupling coefficient between the two degenerated modes can be easily controlled by properly adjusting the dimensions of the cut metallic area (see Figure 17). As a result, the patch radiates with two orthogonal linear polarizations. At the frequency where the phase difference between the two orthogonal polarizations equals 90 , a circular polarized wave is radiated. Also, the excitation of the two resonances can be used to increase the operational bandwidth of the antenna, following the techniques described in the work of Alvarez-Melcón and Mosig [1998].

[45] It is also worth mentioning that for this structure care must be taken to properly analyze the discontinuity presented by the input feeding line embedded inside the dielectric substrate. Owing to the surface charge induced on the metallic line, the normal component of the electric flux density $(\bar{D})$ in the dielectric will present a discontinuity above and below the line. It is important to take into account this fact, since then the volume brick cells touching the input feeding line can not form a single basis function (since this will impose a wrong continuity condition for $\bar{D}$ ). Instead, these cells must be finished on distinct half-rooftop functions, each one taking care of 


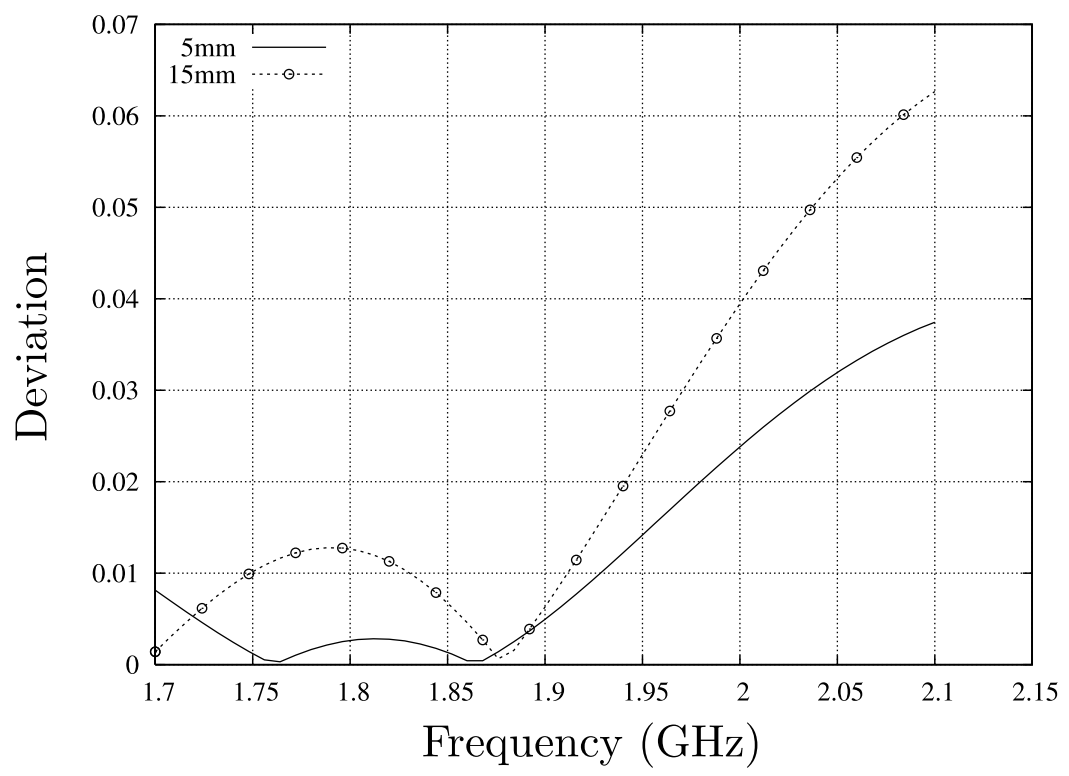

Figure 13. Relative deviation when different cell sizes are used, with respect to the nominal situation (cell size of $10 \mathrm{~mm}$ ).

the different value of above and below the line. In the software developed, we have extended the geometrical treatment of the structures to account for this situation. Once a metallic area is placed inside a dielectric, a search mechanism is started to find the volume brick cells in touch with the metallic area. Once identified, two half-rooftop functions are defined on them (instead of defining the standard single full rooftop function).

[46] In Figure 18 we present measured and simulated results for the reflection coefficient of the antenna obtained for the structure in Figure 17, showing the two degenerated coupled resonances of the patch. By properly coupling both resonances, a relatively large operational bandwidth of $6.2 \%$ has been obtained, measured at $\left|S_{11}\right|=10 \mathrm{~dB}$. In the figure we present the measured results together with theoretical predictions obtained with an infinite-size dielectric model and with the new IE technique for finite-size dielectric substrates. It can be seen that the agreement is in all cases good.

[47] In Figure 19 we further present the input impedance of the antenna. Again measured and simulated results obtained with the infinite and finite-size models are in good agreement. Finally, Figure 20 presents the results obtained with the infinite and finite-size dielectric models for the axial ratio at the center frequency of the operational bandwidth, in the $\mathrm{E}$ plane $(\mathrm{H}$ plane shows similar behavior). It can be seen that the agreement for elevation angles between $-90^{\circ}$ and $90^{\circ}$ is good. For all other directions, the axial ratio is shown only for the finite size model, since the infinite one predicts no radiation in this area of the space. Also the measured result at broadside is shown with a star symbol in the same graphic. It can be seen that a good circular polarized wave is radiated at this frequency, with values of axial ratio lower than $2 \mathrm{~dB}$ close to broadside.

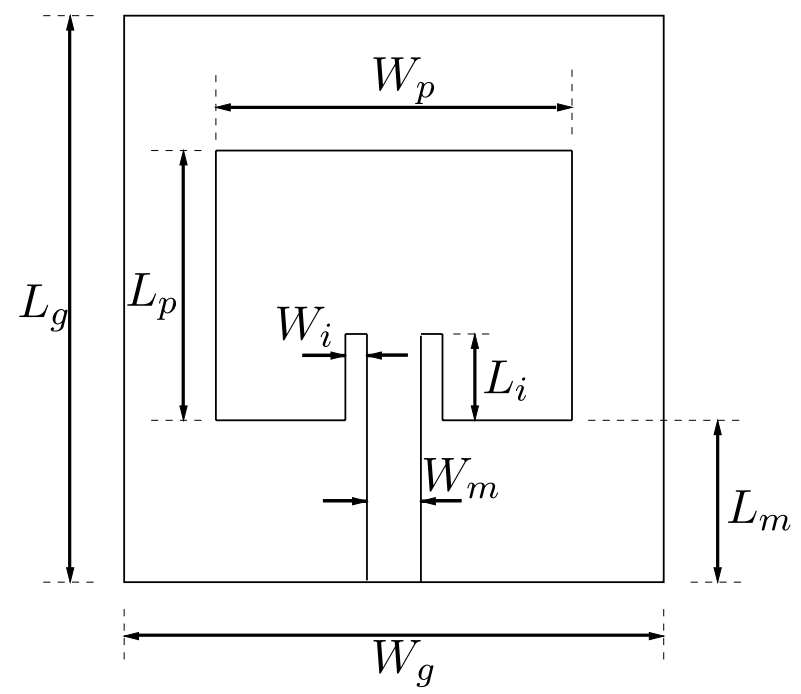

Figure 14. Printed patch antenna introduced in the work of Tiezzi et al. [2000a]. The dimensions are $L_{g}=$ $40.0 \mathrm{~mm}, W_{g}=40.0 \mathrm{~mm}, L_{p}=15.70 \mathrm{~mm}, W_{p}=24.0 \mathrm{~mm}$, $L_{m}=13.0 \mathrm{~mm}, W_{m}=4.90 \mathrm{~mm}, L_{i}=3.30 \mathrm{~mm}$, and $W_{i}=$ $0.55 \mathrm{~mm}$. The dielectric permittivity of the substrate is $\varepsilon_{r}=2.33$, and the thickness $h=1.57 \mathrm{~mm}$. 


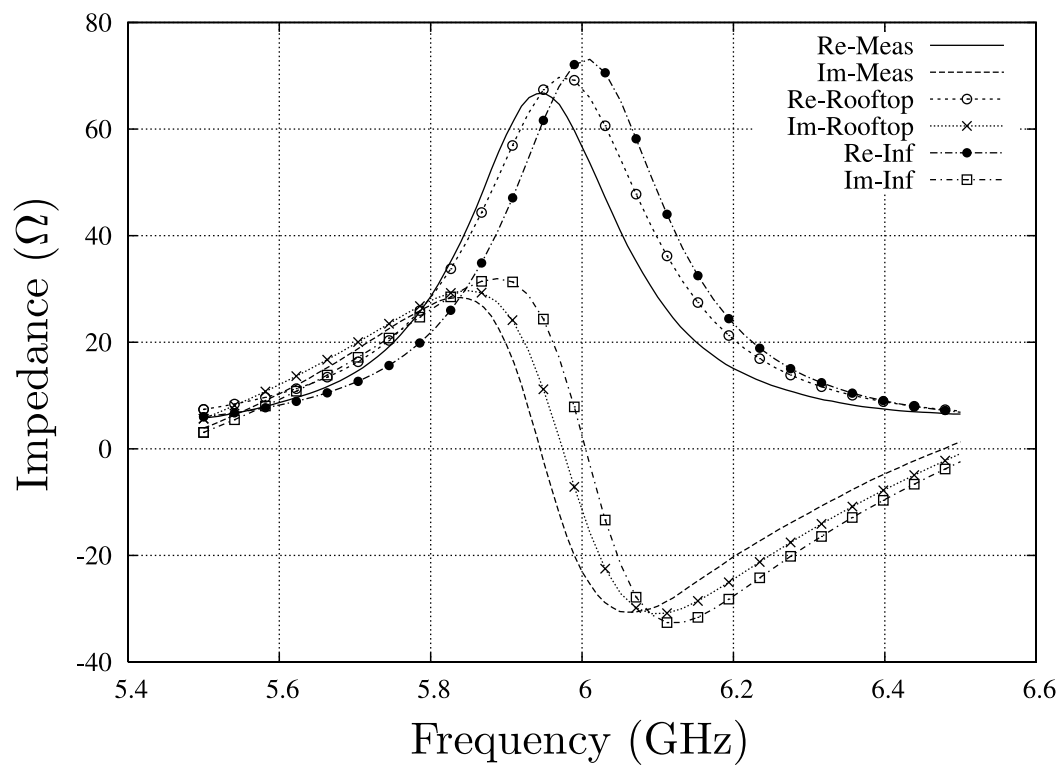

Figure 15. Input impedance of the structure shown in Figure 14.

[48] The problem of the slow convergence associated to the partial overlapping of the cells, mentioned in section 6 , has been studied for the antenna shown in Figure 9. We have performed two different studies on this antenna. The first one, shown in Figure 21a, presents the reflection coefficient of the antenna under four different conditions. The first curve in solid line uses $(10 \times 10)$ cells in the dielectric (and one cell along its

$$
(\varphi=0)
$$

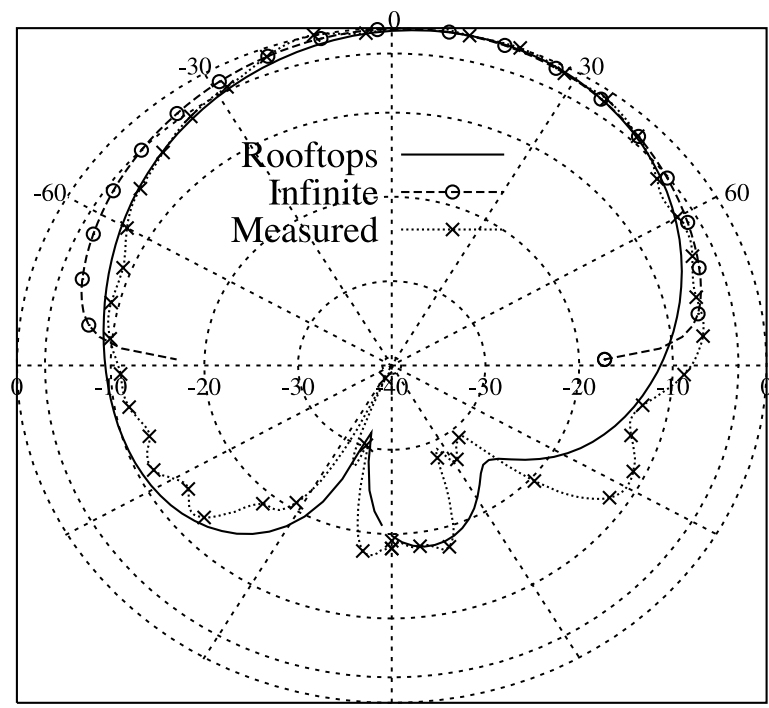

a) E-Plane (dB)

$$
(\varphi=\pi / 2)
$$

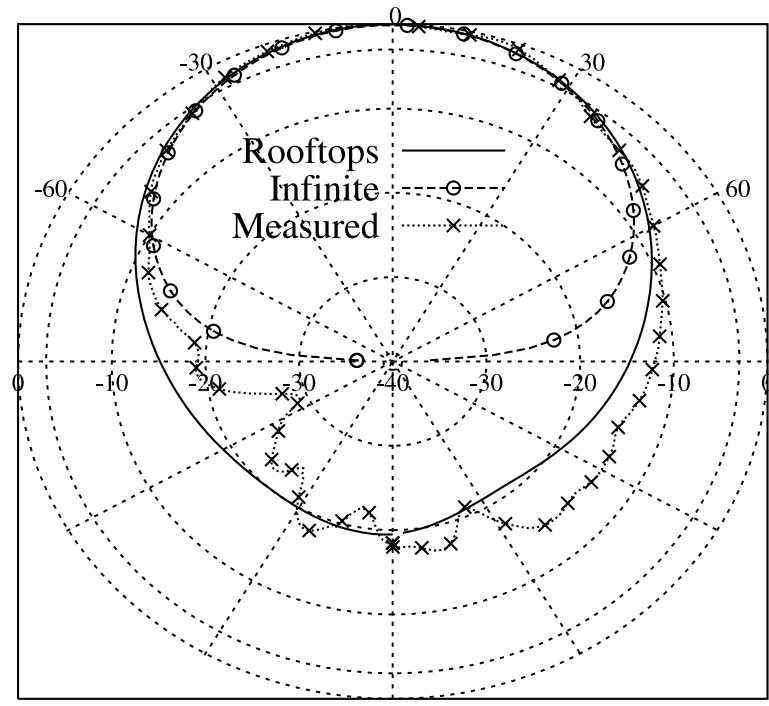

b) H-Plane (dB)

Figure 16. Radiation patterns of the antenna shown in Figure 14. 

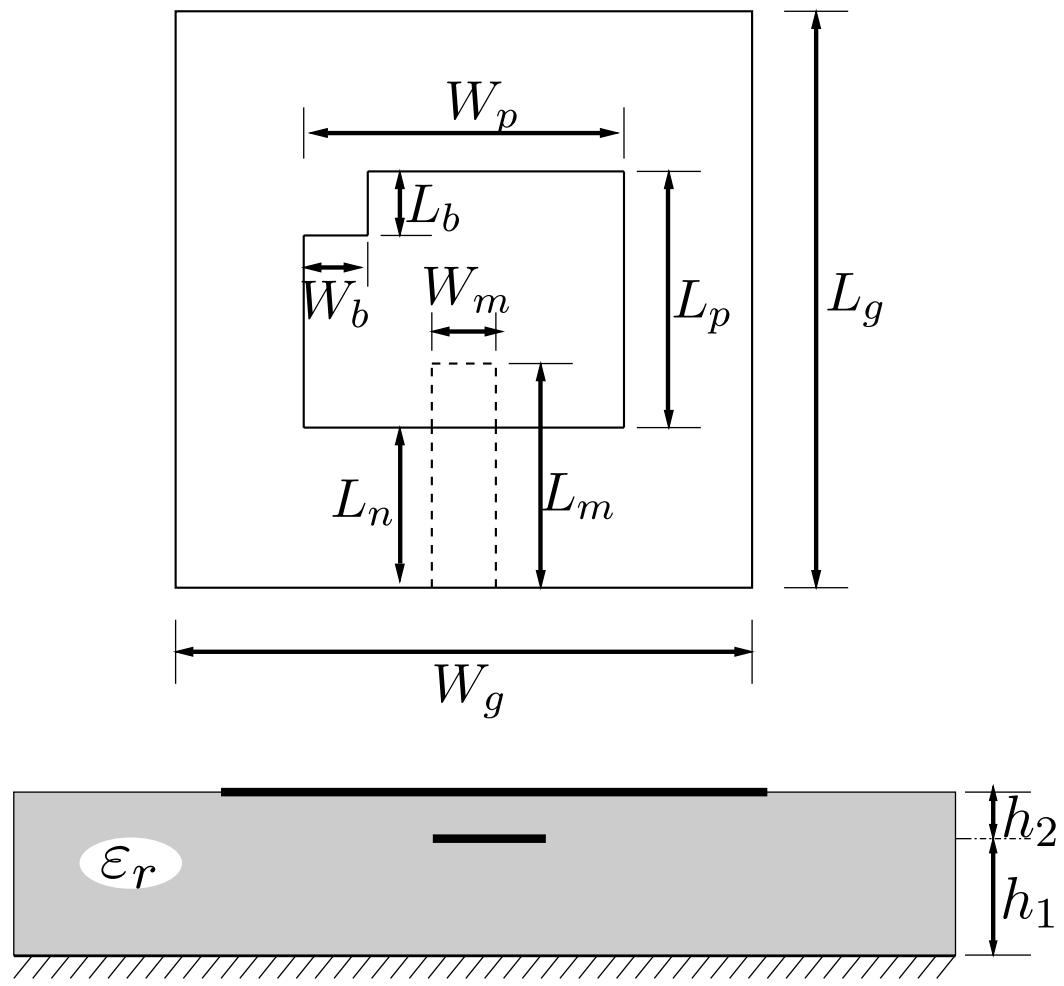

Figure 17. Circular polarized multilayer printed antenna: $W_{p}=20.4 \mathrm{~mm}, L_{m}=16 \mathrm{~mm}, W_{m}=$ $3.0 \mathrm{~mm}, L_{n}=10.0 \mathrm{~mm}, L_{b}=3.7 \mathrm{~mm}, W_{b}=3.7 \mathrm{~mm}, \varepsilon_{r}=2.33, h_{1}=1.57 \mathrm{~mm}$, and $h_{2}=0.51 \mathrm{~mm}$.

height $)$, and $(15 \times 15)$ cells in the metallic areas, therefore leading to a partial overlapping situation. The second curve with cross symbols uses $(15 \times 15)$ cells in both the dielectric and in the metallic areas, therefore avoiding partial overlapping. The third curve with white circles uses $(25 \times 25)$ cells in the dielectric, while maintaining $(15 \times 15)$ cells in the metallic areas to produce again partial overlapping. Finally, the fourth curve with black circles uses $(30 \times 30)$ cells in both the dielectric and the metallic areas, therefore avoiding again the partial overlapping situation. As it can be seen in the figure, the effect of the partial overlapping is a frequency shift toward higher frequencies of the antenna transfer function. Indeed, the results with $(10 \times 10)$ shows a frequency shift of about $300 \mathrm{MHz}$ with respect to the results obtained under no partial overlapping situation $(15 \times 15$ and $30 \times 30)$. The results with $(25 \times 25)$ contains again a partial overlapping situation, and a frequency shift of about $100 \mathrm{MHz}$ can be observed. It can be seen that the frequency shift this time is smaller than before. This is due to the fact that the averaging process that takes place for the boundary conditions of the normal components of the fields, introduces small errors when the averaged cells are small in size. Finally, the results with $(15 \times 15)$ and

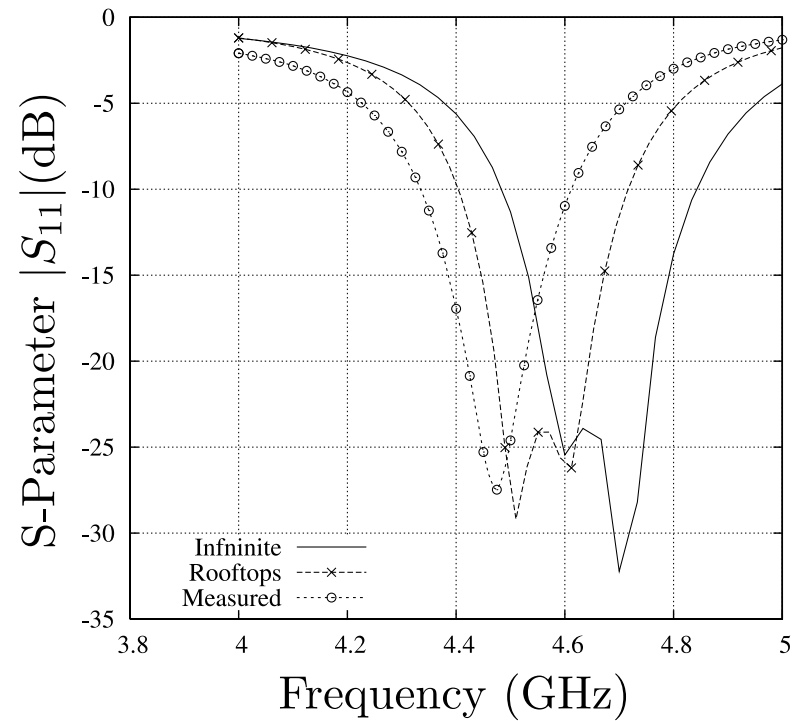

Figure 18. Measured and simulated reflection coefficient for the circular polarized antenna shown in Figure 17. Results with the infinite, and finite size dielectric models are included. 


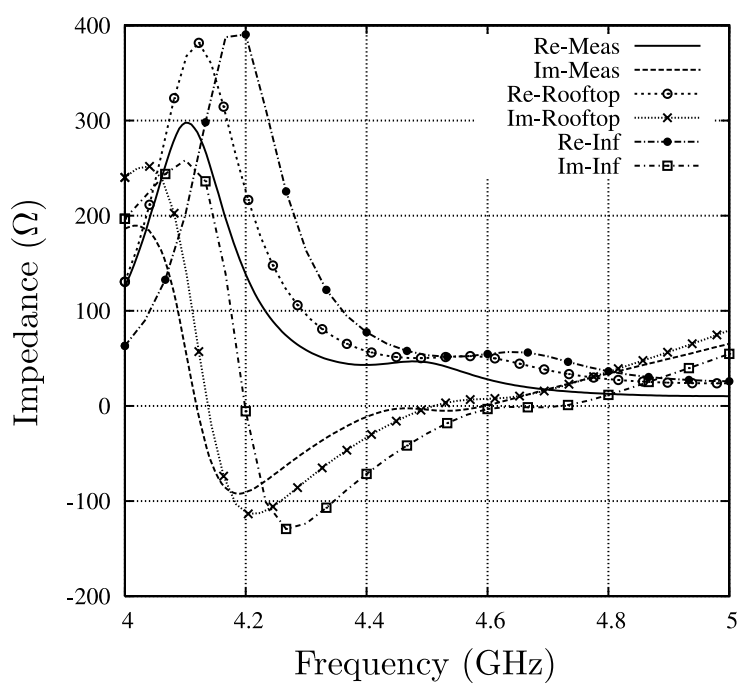

(a) Rectangular plot.

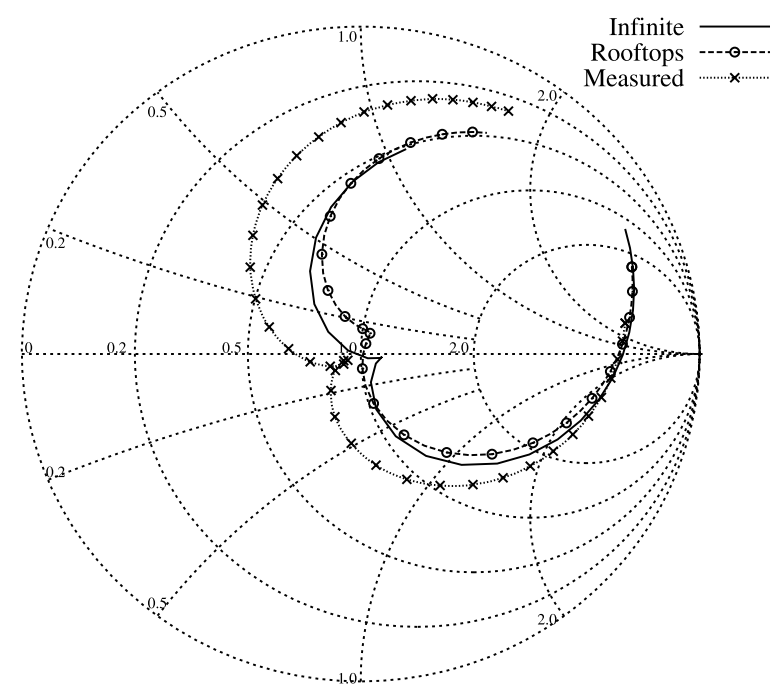

(b) Smith Chart.

Figure 19. Measured and simulated input impedance of the antenna shown in Figure 17. Results with the infinite and finite size dielectric models are included.

$(30 \times 30)$ are very similar, since there are no partial overlapping in them. The small differences between them are due to a different refinement of the meshes.

[49] In order to see how fast a partial overlapping situation converges to a nonpartial overlapping situation, we have performed a second study shown in Figure $21 \mathrm{~b}$. In this study the metallic areas are always discretized with $(15 \times 15)$ cells, while a different number of cells are used for the discretization of the dielectric substrate. Consequently, the $(15 \times 15)$ curve (cross symbols) shows the results without partial overlapping. The curve with $(10 \times 10),(25 \times 25)$ and $(40 \times 40)$ cells represent situations with full partial overlapping. Finally, the curve with $(30 \times 30)$ cells (black circles) represents a very interesting situation, where a partial overlapping of order two takes place between the cells in the metallic areas and those of the dielectric substrate. We have defined a partial overlapping of order two, when an integer number of dielectric cells lays inside a single cell of a metallic area. We can see from the results that again partial overlapping produces a frequency shift in the antenna response. It is interesting to observe that for the case of partial overlapping of order two, the frequency shift is smaller that when a full partial overlapping takes place. This is even the case for the results obtained with $(40 \times 40)$ cells. In spite of using more cells in the dielectric, the frequency shift obtained is larger than for the case of a partial overlapping of order two $(30 \times 30$ cells $)$.
[50] It can be seen from the results that although the partial overlapping between cells has some impact on the numerical accuracy of the method, the negative effect can be reduced by refining the mesh in the dielectric substrate. This is an important conclusion of the technique developed, which allows for an easy and flexible

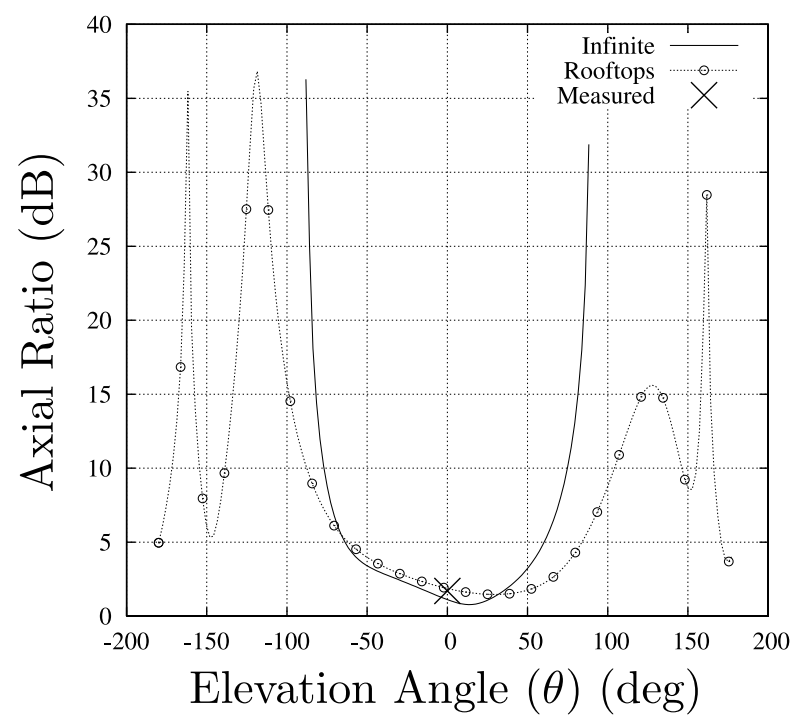

Figure 20. Axial ratio obtained with the infinite and finite size dielectric models. Measured result at broadside is shown by the "X." 


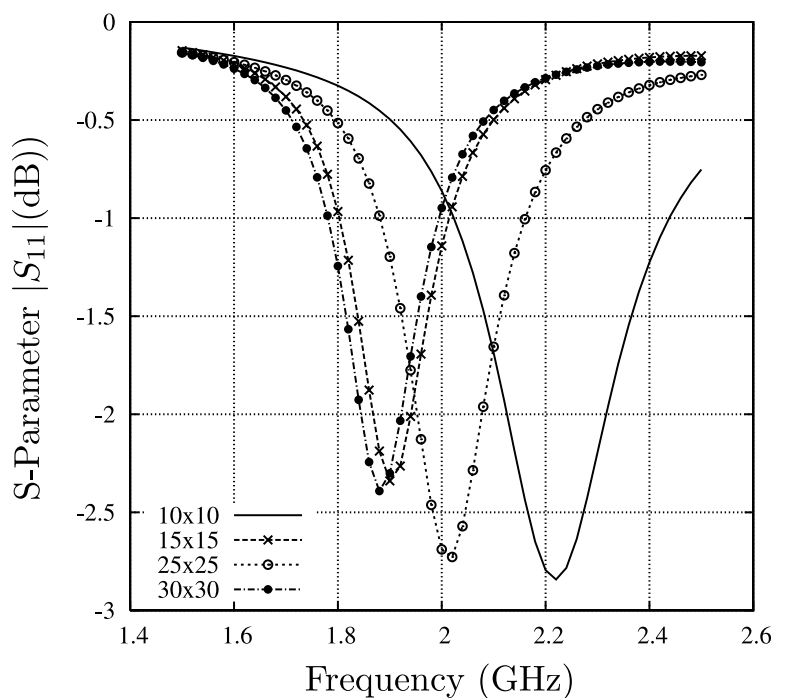

(a) Two different numbers of cells in metallic areas (15x15 and 30x30 cells).

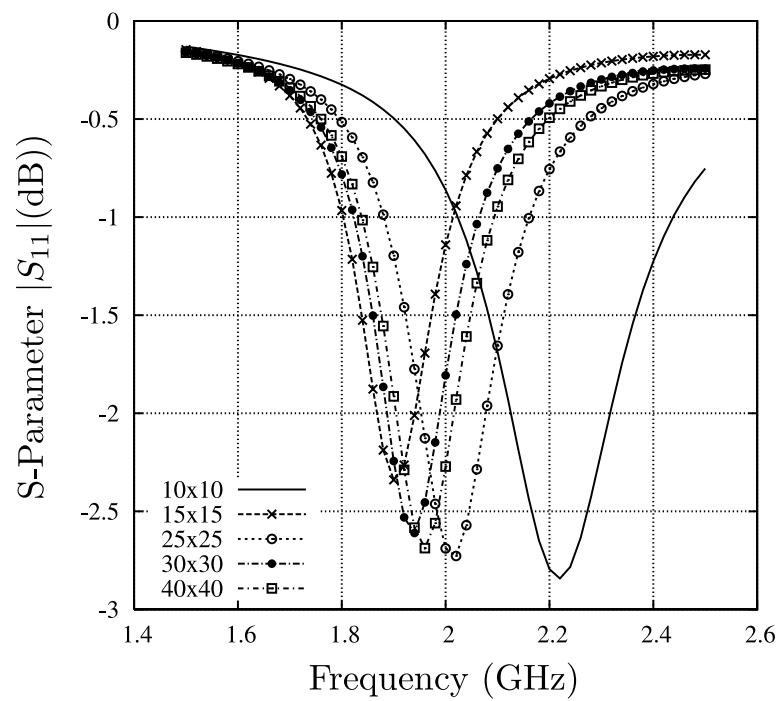

(b) Constant number of cells in metallic areas (15x15 cells).

Figure 21. Partial overlapping study between dielectric bodies and metallic area meshes.

discretization of the metallic areas and dielectric objects, which can now be discretized in a totally independent manner.

\section{Conclusions}

[51] In this paper we have presented two efficient numerical implementations of the volume equivalent integral equation technique. In addition, a novel excitation model has been proposed for finite ground plane structures. The novel technique allows to define different mesh densities on the ground plane and on the input feeding line of printed circuits and antennas. The new models have been applied to the calculation of the electrical characteristics of finite-size microstrip structures. Both the input impedance of microstrip patch antennas and the radiation patterns are in good agreement as compared to measurements. The new technique can be used for the design of microstrip circuits with reduced volume, and for the prediction of the backside radiation of real practical microstrip antennas. A novel procedure has been implemented to allow for a partial overlapping to occur between the cells used in the discretization of the dielectric bodies, and those used in the discretization of the metallic areas. The technique has shown to be useful if a limited impact is allowed in the numerical precision of the results. The main advantage of using the novel technique combined with partial overlapping of the meshes, is that dielectric bodies and printed metallic areas can be meshed independently. This simplifies considerably the mesh generation and subsequent geometrical treatment of complex printed circuits.

[52] Acknowledgment. This work has been supported by the Spanish National Project ESP2001-4546-PE, and Regional Seneca Project PB/4/FS/02.

\section{References}

Aksun, M. I., and G. Dural (1996), Comparative evaluation of absorbing boundary conditions using Green's functions for layered media, IEEE Trans. Antennas Propag., 44(2), $152-156$.

Alvarez-Melcón, A., and J. R. Mosig (1998), Strip, slot, air, inverted patch (SSAIP): A cavity backed alternative to broadband communication antennas, Radio Sci., 33(6), $1525-1542$.

Anderson, E., et al. (1999), LAPACK Users' Guide, 3rd ed., Soc. for Indust. and Appl. Math., Philadelphia, Penn.

Bokhari, S. A., J. R. Mosig, and F. E. Gardiol (1992), Radiation pattern computation of microstrip antennas on finite size ground planes, IEE Proc., 139, 278-286.

Bunger, R., and F. Arndt (1997), Efficient mpie approach for the analysis of three-dimensional microstrip structures in layered media, IEEE Trans. Microw. Theory Tech., 45(8), $1141-1153$.

Catedra, M., E. Gago, and L. Nuño (1989), A numerical scheme to obtain the rcs of three-dimensional bodies of resonant size using the conjugate gradient method and the fast fourier transform, IEEE Trans. Antennas Propag., 37, $528-537$. 
Chew, W. C., J.-M. Jin, E. Michielssen, and J. Song (2001), Fast and Efficient Algorithms in Computational Electromagnetics, chap. 11, pp. 487-537, Artech House, Norwood, Mass.

Cools, R. (1999), Monomial cubature rules since stroud: A compilation, part 2, J. Comput. Appl. Math., 112(1-2), $21-27$

Eleftheriades, G. V., and J. R. Mosig (1996), On the network characterization of planar passive circuits using the method of moments, IEEE Trans. Microw. Theory Tech., 44(3), $438-445$.

Gay-Balmaz, P., and J. R. Mosig (1997), Three-dimensional planar radiating structures in stratified media, Int. J Microw. Millimeter Wave Comput. Aided Eng., 7, 330-343.

Gedney, S., and C. C. Lu (2003), High-order integral equation solution based on a hybrid volume/surface formulation, paper presented at 19th Annual Review of Progress in Applied Computational Electromagnetics, Appl. Comput. Electromagn., Monterey, Calif.

Kinayman, N., and M. I. Aksun (1997), Efficient use of closed form green's functions for the analysis of planar geometries with vertical connections, IEEE Trans. Microw. Theory Tech., 45(5), 593-603.

Lu, C. C., and W. C. Chew (2000), A coupled surface-volume integral equation approach for the calculation of electromagnetic scattering from composite metallic and material target, IEEE Trans. Antennas Propag., 48(12), 1866-1868.

Lu, C. C., and C. Yu (2002), Analysis of microstrip structures of finite ground plane using the hybrid volume-surface integral equation approach, paper presented at Antennas and Propagation Intenational Symposium, Inst. of Electr. and Electron. Eng., San Antonio, TX.

Melcon, A. A., J. R. Mosig, and M. Guglielmi (1999), Efficient $\mathrm{CAD}$ of boxed microwave circuits based on arbitrary rectangular elements, IEEE Trans. Microw. Theory Tech., 47(7), $1045-1058$.

Mesa, F., and R. Marques (1995), Integral representation of spatial Green's function and spectral domain analysis of leaky covered strip-like lines., IEEE Trans. Microw. Theory Tech., 43(4), 828-837.

Michalski, K. A., and D. Zheng (1990), Electromagnetic scattering and radiation by surfaces of arbitrary shape in layered media, part i: Theory, IEEE Trans. Antennas Propag., 38(3), 335-344.

Mosig, J. R. (1988), Arbitrarily shaped microstrip structures and their analysis with a mixed potential integral equation, IEEE Trans. Microw. Theory Tech., 36(2), 314-323.

Mosig, J. R., and F. E. Gardiol (1982), A Dynamic Radiation Model for Microstrip Structures, chap. 3, pp. 139-237, Elsevier, New York.

Sarkar, T. K., and E. Arvas (1990), An integral equation approach to the analysis of finite microstrip antennas: Volume/surface formulation., IEEE Trans. Antennas Propag., 38(3), 305-312.

Sarkar, T. K., S. M. Rao, and A. R. Djordjevic (1990), Electromagnetic scattering and radiation form finite microstrip structures, IEEE Trans. Microw. Theory Tech., 38(11), $1568-1575$.

Schaubert, D. H., D. R. Wilton, and A. W. Glisson (1984), A tetrahedral modeling method for electromagnetic scattering by arbitrarily shaped inhomogeneous dielectric bodies, IEEE Trans. Antennas Propag., 32(1), 77-85.

Tavakkol-Hamedani, F., A. Tavakoli, and L. Shafai (2002), Analysis of finite-microstrip structures using surface equivalence principle and multiple network theory (SEMN), IEEE Trans. Antennas Propag., 50(8), 1128-1137.

Tiezzi, F., A. A. Melcon, and J. R. Mosig (2000a), A new excitation model for microstrip antennas on finite size ground planes, paper presented at IEEE AP-2000 Symposium, Eur. Space Ag., Danos, Switzerland.

Tiezzi, F., A. A. Melcon, and J. R. Mosig (2000b), A new excitation model for probe-fed printed antenna on finite size ground planes, Appl. Computat. Electromagn. Soc., 15(2), $115-125$.

A. Alvarez Melcon, D. Cañete Rebenaque, J. L. Gomez Tornero, J. Pascual Garcia, and F. Quesada Pereira (corresponding author), Department of Information Technologies and Communications, Technical University of Cartagena, Campus Muralla del Mar s/n, E-30202 Cartagena (Murcia), Spain. (fernando.quesada@upct.es) 